\title{
Neutrino splitting for Lorentz-violating neutrinos: Detailed analysis
}

\author{
G. Somogyi, ${ }^{1, *}$ I. Nándori, ${ }^{1,2,3}$ and U. D. Jentschura ${ }^{1,3,4, \dagger}$ \\ ${ }^{1}$ MTA-DE Particle Physics Research Group, P.O. Box 51, H-4001 Debrecen, Hungary \\ ${ }^{2}$ University of Debrecen, P.O. Box 105, H-4010 Debrecen, Hungary \\ ${ }^{3}$ MTA Atomki, P.O. Box 51, H-4001 Debrecen, Hungary \\ ${ }^{4}$ Department of Physics, Missouri University of Science and Technology, Rolla, Missouri 65409, USA
}

(Received 22 December 2018; published 29 August 2019)

\begin{abstract}
Lorentz-violating neutrino parameters have been severely constrained on the basis of astrophysical considerations. In the high-energy limit, one generally assumes a superluminal dispersion relation of an incoming neutrino of the form $E \approx|\vec{p}| v$, where $E$ is the energy, $\vec{p}$ is the momentum and $v=\sqrt{1+\delta}>1$. Lepton-pair creation due to a Cerenkov-radiation-like process $\left(\nu \rightarrow \nu+e^{-}+e^{+}\right)$becomes possible above a certain energy threshold, and bounds on the Lorentz-violating parameter $\delta$ can be derived. Here, we investigate a related process, $\nu_{i} \rightarrow \nu_{i}+\nu_{f}+\bar{\nu}_{f}$, where $\nu_{i}$ is an incoming neutrino mass eigenstate, while $\nu_{f}$ is the final neutrino mass eigenstate, with a superluminal velocity that is slightly slower than that of the initial state. This process is kinematically allowed if the Lorentz-violating parameters at high energy differ for the different neutrino mass eigenstates. Neutrino splitting is not subject to any significant energy threshold condition and could yield quite a substantial contribution to decay and energy loss processes at high energy, even if the differential Lorentz violation among neutrino flavors is severely constrained by other experiments. We also discuss the $S U(2)_{L}$-gauge invariance of the superluminal models and briefly discuss the use of a generalized vierbein formalism in the formulation of the Lorentz-violating Dirac equation.
\end{abstract}

DOI: $10.1103 /$ PhysRevD.100.035036

\section{INTRODUCTION}

A possible Lorentz violation in the neutrino sector has been the subject of intense investigations in recent years, with a rich texture of interesting models and corresponding scenarios having been explored in the literature (see Refs. [1-9]). The apparent cutoff of the neutrino spectrum seen by IceCube at a threshold energy $E=E_{\text {th }} \approx 2 \mathrm{PeV}$ [10-12] has given rise to interesting speculations, which include rather stringent limits on Lorentz-violating parameters $[8,13,14]$.

Due to their different masses, neutrino decays between generations are in fact kinematically allowed, but for neutrinos which fulfill a Lorentz-invariant dispersion relation, the decays are excessively long and exceed the age of the Universe by orders of magnitude. Namely, in addition to the small magnitude of the mass differences, the Glashow-Iliopoulos-Maiani (GIM) mechanism [15,16]

\footnotetext{
gabor.somogyi@cern.ch ulj@mst.edu
}

Published by the American Physical Society under the terms of the Creative Commons Attribution 4.0 International license. Further distribution of this work must maintain attribution to the author(s) and the published article's title, journal citation, and DOI. Funded by SCOAP . leads to cancellations between generations. For quarks, the GIM mechanism relies on the unitarity of the CabibboKobayashi-Maskawa matrix, whereas for neutrinos, one assumes unitarity of the Pontecorvo-Maki-NakagawaSakata (PMNS) matrix. However, under a (small) Lorentz violation, additional decay channels exist which are not GIM suppressed and lead to decay of a neutrino without changing the flavor or mass eigenstates. Among these, two decay and energy loss mechanisms have been given special attention, namely, vacuum pair emission (VPE), which corresponds to lepton-pair Cerenkov radiation (LPCR, $\nu \rightarrow \nu+e^{-}+e^{+}$), and neutrino splitting, i.e., neutrino-pair Cerenkov radiation (NPCR, $\nu_{i} \rightarrow$ $\nu_{i}+\nu_{f}+\bar{\nu}_{f}$ ). We will use both designations for each process interchangeably in the current article.

Concerning NPCR, it has been pointed out at various places in the literature $[8,17,18]$ that this process should be suppressed, because all neutrino flavors are known to propagate at approximately the same velocity. The papers $[17,19]$ cite bounds on the relative difference of the velocities of neutrino mass eigenstates on the level of $10^{-19}$, based on the argument that otherwise, the interference pattern of the neutrino oscillations would be smeared. The short-baseline experiment [19] has the advantage that the entire beamline is under laboratory control. The paper [20] gives stricter bounds on the order of $10^{-22}$, also from 
short-baseline experiments. According to Ref. [21], the IceCube collaboration has obtained, for the $\mu-\tau$ neutrino sector, even stricter bounds on the order of $10^{-27}$, coming from the survival of atmospheric muon neutrinos in the energy range $100 \mathrm{GeV}$ to $10 \mathrm{TeV}$, assuming maximal mixing for some of the neutrino flavor eigenstates.

If all neutrino propagation velocities are precisely equal to each other, then the NPCR process is kinematically forbidden [8] in the Lorentz-violating Standard Model extension, and it seems that theoretical studies have thus focused on Planck-scale operators inducing the NPCR decay. These typically entail formulas for the decay rate which scale with the eighth and higher powers of the neutrino energy [see Eqs. (19) and (20) of Ref. [8] ]. The corresponding $\delta$ parameters are proportional to the inverse of the Planck mass for dimension-five operators [see Eqs. (6) and (10) of Ref. [8], the latter with $n=1$ ] and proportional to the inverse of the square of the Planck mass for dimension-six operators [see Eqs. (6) and (10) of Ref. [8], the latter with $n=2$, as well as Refs. [18,22]]. On one hand, one can argue that a detailed analysis of the NPCR process within the Lorentz-violating Standard Model extension is thus superfluous. On the other hand, the NPCR process is unique among the decay processes for superluminal neutrinos in that the energy threshold is negligible [8]. It could thus be worthwhile to augment several treatments, recorded in the literature [23-25], by a detailed calculation of the effect, in the spirit of Ref. [17], which includes an analysis of the model dependence.

In order to put things into perspective, let us recall that the VPE (or LPCR) decay rate is proportional to $[17,26]$

$$
\Gamma_{\nu \rightarrow \nu e^{-} e^{+}} \propto G_{F}^{2} E^{5}\left(\delta_{\nu}-\delta_{e}\right)^{3},
$$

where $\delta_{\nu}=v_{\nu}^{2}-1 \approx 2\left(v_{\nu}-1\right)$ is the Lorentz-violating parameter of the incoming neutrino (of energy $E$ ), and $\delta_{e}$ is the corresponding parameter for the electron-positron pair. Note that the $v_{\nu}$ parameter here takes the role of a maximum achievable velocity, consistent with the models proposed in Refs. [17,26]. (Note that units with $\hbar=c=$ $\epsilon_{0}=1$ are used throughout this paper.) The corresponding parameters in Refs. [14,8] are defined for the deviations $v_{\nu}-1$ and $v_{e}-1$ and thus differ from ours by a factor 2 . The energy loss rate is found to be $[17,26]$

$$
\frac{\mathrm{d} E}{\mathrm{~d} x} \propto G_{F}^{2} E^{6}\left(\delta_{\nu}-\delta_{e}\right)^{3}
$$

Canonically $[8,14]$, one then makes the additional assumption that $\delta_{e}=0$ and justifies this on account of the known subluminal nature of the electron at low energies. Furthermore, as pointed out in Refs. [13,14], one has a bound on the order of $\delta_{e} \leq 1.04 \times 10^{-20}$ for the Lorentz-violating parameter in the electron-positron sector.
(The bound is actually given as $0.52 \times 10^{-21}$ in Refs. $[13,14]$, but we again recall the additional factor 2 .)

If one assumes that $\delta_{e}>0$ could also be superluminal for electrons in the high-energy region, then the flavorindependent $\delta_{\nu}$ for neutrinos could be as large as $\delta_{\nu} \approx 2.0 \times 10^{-20}$, and still be compatible with the bound on neutrinos from astrophysics, and with the bounds on the electron (see p. 7 of Ref. [8]).

In Ref. [8], the NPCR process in the formulation adopted here was simply discarded on the basis of the argument that it vanishes when all neutrinos propagate at the same speed. We still found it interesting to carry out a more detailed analysis of the NPCR, in order to map out a possible role of this effect in the analysis of astrophysical data.

Based on an obvious analogy with Eq. (1), one might expect a functional form

$$
\Gamma_{\nu_{i} \rightarrow \nu_{i} \nu_{f} \bar{\nu}_{f}} \propto G_{F}^{2} E^{5}\left(\delta_{i}-\delta_{f}\right)^{3},
$$

for the NPCR-induced decay rate, where $\delta_{i}$ and $\delta_{f}$ refer to the Lorentz-violating parameters of the initial and final states. However, we can anticipate here that one also finds terms of the form

$$
\Gamma_{\nu_{i} \rightarrow \nu_{i} \nu_{f} \bar{\nu}_{f}} \propto G_{F}^{2} E^{5}\left(\delta_{i}-\delta_{f}\right)\left(\delta_{i}+\delta_{f}\right)^{2},
$$

which could play a much more prominent role in the analysis of astrophysical data than previously thought, because the NPCR process essentially has a negligible threshold. (This assumption is made in Ref. [8] and here.) For the energy loss rate, we also find terms of the form

$$
\frac{\mathrm{d} E}{\mathrm{~d} x} \propto G_{F}^{2} E^{6}\left(\delta_{i}-\delta_{f}\right)\left(\delta_{i}+\delta_{f}\right)^{2},
$$

which could also be relevant in the high-energy region.

Some remarks on the gauge structure of the models employed in Refs. $[17,26]$ are in order. Of course, a priori, one would like to preserve the $S U(2)_{L} \times U(1)_{Y}$ gauge structure as much as possible upon the introduction of the Lorentz-breaking parameters. In addition, an inspection of the Lagrangian of the Standard Model extension (SME) [see Eqs. (9) and (10) of Ref. [1] ] reveals that the SME is based on the assumption that the entire gauge structure of the Standard Model is preserved upon the introduction of the Lorentz-violating terms.

In terms of the formulation of the vacuum pair emission $[17,26]$, one faces a certain dilemma: On one hand, if the charged fermions and the neutrinos are grouped in an $S U(2)_{L}$ doublet, and if the Lorentz-breaking parameters are assigned uniformly over all generations, then both NPCR and LPCR decays are kinematically forbidden in the Lorentz-violating Standard Model extension. On the other hand, there are good reasons (with both theoretical and experimental motivations) for the assumption that Lorentz 
violation, if it exists, should be confined to the neutrino sector, without affecting the charged fermions.

Hence, Cohen and Glashow, as well as Bezrukov and Lee [17,26] (see also Sec. VII C 2 of Ref. [4]), chose interaction Lagrangians which, as a closer inspection reveals (see also Appendixes A and B, Sec. VII C 2 of Ref. [4], and Ref. [27]), are not gauge invariant with respect to the full $S U(2)_{L} \times U(1)_{Y}$ gauge group. We here follow the same approach, and, in addition to the analysis of the NPCR decay, attempt to find certain interpolating formulas connecting the models used in Refs. $[17,26]$. Some of the models used by Bezrukov and Lee [see the cryptic remark on "gauge invariance" near Eq. (4) of Ref. [17] ] preserve the gauge structure of the electroweak interaction at least in part (see also Ref. [27]).

The models used in Refs. [17,26] and here are based on two assumptions, namely, (i) that Lorentz violation is confined to the neutrino sector, and that (ii) the effective Fermi theory, possibly with some modifications, still holds for the description of the decay and energy loss processes. We here follow this approach, which was also used in Sec. VII C 2 of Ref. [4], and accept a [perturbative (see Appendix B)] violation of $S U(2)_{L}$ gauge invariance as a price for the attractive Lorentz-violating kinematic scenario under investigation.

This paper is organized as follows. In Sec. II, we discuss the framework of the calculation, partially outlined above, in greater detail. The calculation of the decay and energy loss rates is performed in Sec. III, while results for the rates are presented and bounds on the Lorentz-violating parameters are derived in Sec. IV. Conclusions are reserved for Sec. V.

\section{FRAMEWORK}

\section{A. Theoretical basis and assumptions}

The theoretical basis for the description of the Lorentzviolating states is given by generalized Dirac equations, which contain Lorentz-violating terms. In typical cases [see, e.g., Eq. (1) of Ref. [17]], these give rise to a dispersion relation $E=|\vec{p}| v$ with a Lorentz-violating parameter $v>1$. In the following, we parametrize the departure of $v$ from unity by setting $v=\sqrt{1+\delta}$.

If the virtuality of the incoming superluminal neutrino is large enough,

$$
E^{2}-\vec{p}^{2} \geq\left(2 m_{e}\right)^{2},
$$

then electron-positron pair production becomes possible. This translates into an energy threshold

$$
E_{\mathrm{th}}=\frac{2 m_{e}}{\sqrt{\delta}}, \quad \delta=v^{2}-1,
$$

for the onset of light lepton pair production, (lepton-pair Cerenkov radiation $[20,26]$ ), as manifest in the reaction

$$
\nu \rightarrow \nu+e^{-}+e^{+}
$$

Notice that Eq. (7) is correct in the small $\delta$ limit up to terms of relative order $\delta$. The exact expression for the energy threshold is $E_{\text {th }}=2 m_{e} \sqrt{1+\delta} / \sqrt{\delta}$.

The process (8) gives rise to both a decay rate and an energy loss rate, for any incoming superluminal neutrino, as illustrated in Eqs. (2) and (3) of Ref. [26]. In choosing our convention for the $\delta$ parameter in Eq. (7), according to $v \approx 1+\delta / 2$, we are consistent with Refs. [17,20,26,28], while Refs. $[8,13,14]$ choose their $\delta$ parameters to be equal to just the difference $v-1$. We here adhere to the conventions chosen in previous calculations of neutrino decay processes, given in Refs. [17,26,28]. In Ref. [26], it was stated that yet another process,

$$
\nu_{i} \rightarrow \nu_{i}+\nu_{f}+\bar{\nu}_{f}
$$

might become kinematically possible for superluminal neutrinos. For other processes limiting superluminal models in the low-energy domain, we refer to Ref. [29]. Both processes in Eqs. (8) and (9) involve the exchange of a $Z^{0}$ boson, as is evident from the diagrammatic representation in Fig. 1. The interesting feature of the process given in Eq. (9) is that the corresponding energy threshold,

$$
E_{\mathrm{th}}=\frac{2 m_{\nu}}{\sqrt{\delta_{i}}}, \quad \delta_{i}=v_{i}^{2}-1,
$$

is smaller than (7) by at least 6 orders of magnitude and can thus safely be ignored [8]. Here, the parameter $\delta_{i}$ describes the Lorentz violation of the initial neutrino mass eigenstate as depicted in Fig. 1. We assume all $\delta$ parameters in this paper to be positive. The parameter $m_{\nu}$ in Eq. (10) denotes the mass of the "final" mass eigenstate denoted as $\nu_{f}=\nu_{f}^{(m)}$ in Fig. 1, assuming a dispersion relation of the

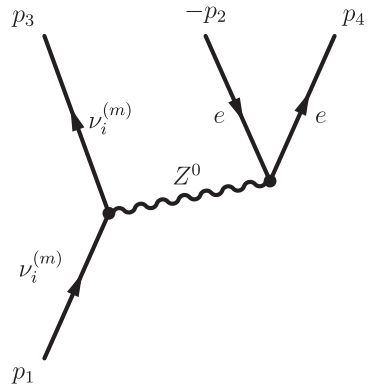

(a)

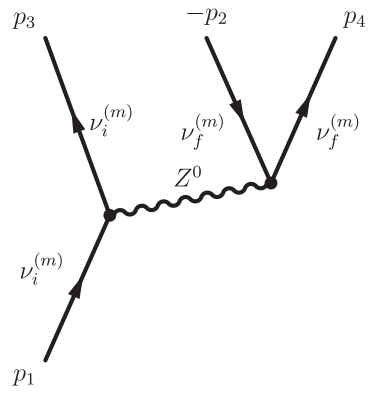

(b)
FIG. 1. The Feynman diagrams for the LPCR (a) and NPCR (b) processes illustrate the exchange of a virtual $Z$ boson. They become kinematically possible for an incoming neutrino mass eigenstate $\left|\nu_{i}^{(m)}\right\rangle$, which decays into a state of the same mass, but lower energy, also labeled $\left|\nu_{i}^{(m)}\right\rangle$, and an electron-positron pair (LPCR) or a neutrino-antineutrino pair (NPCR). 
form given below in Eq. (11). (We shall comment on the suitability of the mass eigenstate basis for the calculation in the following.) For reference, and somewhat pessimistically, we assume that $m_{\nu}$ is the largest among the masses of the neutrino mass eigenstates; even under this assumption, the mass $m_{\nu}$ entering the threshold (10) is still safely smaller than $1 \mathrm{eV}$. In the following, we shall concentrate on the high-energy region, where mass terms in the dispersion relation (11) become irrelevant, and pair production becomes possible for $\delta_{i}>\delta_{f}$, as an investigation of the available phase space for final states shows. We thus investigate a possible additional relevance of neutrino-pair Cerenkov radiation, the process given in Eq. (9), as an additional means of deriving limits on Lorentz-violating parameters for superluminal neutrinos.

To this end, we assume that it is appropriate to generalize the dispersion relation $E=|\vec{p}| v$ to the following form (see Ref. [30] and Appendix A):

$$
E_{k}=\sqrt{\vec{p}^{2} v_{k}^{2}+m_{k}^{2} v_{k}^{4}}
$$

where we consider an (incoming or final) mass eigenstate $k=1,2,3$. Here, the subscript $k=i$ denotes, simultaneously, the initial state of the process, and equivalently a mass eigenstate, while $k=f$ denotes the final state. We know that the masses $m_{i}$ are nonvanishing and different from each other, so that it makes perfect sense [see Eq. (11)] to also consider slightly different Lorentzviolating parameters $v_{i}$, even though their relative differences are very tightly constrained [19-21].

A difficulty arises. Namely, because the weak-interaction Lagrangian is flavor-diagonal, neutrinos are always generated in flavor eigenstates. However, if we assume the Lorentz-violating parameters to be different, then we need to consider the decay process in the basis of mass eigenstates. Let us denote a mass eigenstate by the superscript $(m)$, with eigenstates labeled as $k=1,2,3$, and a flavor eigenstate by the superscript $(f)$, with an eigenstate labeled by the subscript $\ell=1,2,3$. The two are related by the PMNS matrix $U$,

$$
\nu_{k}^{(f)}=\sum U_{k \ell} \nu_{\ell}^{(m)}
$$

For the coupling to a $Z^{0}$ boson, the interaction Lagrangian is

$$
\mathcal{L}=-\frac{g_{w}}{4 \cos \theta_{W}} \sum_{k} \bar{\nu}_{k}^{(f)} \gamma^{\mu}\left(1-\gamma^{5}\right) \nu_{k}^{(f)} Z_{\mu}
$$

where $Z_{\mu}$ denotes the $Z$ boson field, and the $\gamma^{\mu}$ are the usual Dirac matrices, while $\gamma^{5}$ is the fifth current Dirac matrix. The weak coupling constant is $g_{w}$, and $\theta_{W}$ is the Weinberg angle. We can reformulate Eq. (13) as
$\mathcal{L}=-\frac{g_{w}}{4 \cos \theta_{W}} \sum_{k, \ell, \ell^{\prime}} U_{\ell k}^{+} U_{k \ell^{\prime}} \bar{\nu}_{\ell}^{(m)} \gamma^{\mu}\left(1-\gamma^{5}\right) \nu_{\ell^{\prime}}^{(m)} Z_{\mu}$,

where all summation indices cover the range $k, \ell, \ell^{\prime}=$ $1,2,3$. Assuming unitarity of the PMNS matrix, $\sum_{k} U_{\ell k}^{+} U_{k \ell^{\prime}}=\delta_{\ell \ell^{\prime}}$, one arrives at

$$
\mathcal{L}=-\frac{g_{w}}{4 \cos \theta_{W}} \sum_{\ell} \bar{\nu}_{\ell}^{(m)} \gamma^{\mu}\left(1-\gamma^{5}\right) \nu_{\ell}^{(m)} Z_{\mu},
$$

exhibiting diagonality in the mass eigenstate basis. We can thus variously choose to evaluate the decay, and energy loss, rates, in the basis of flavor eigenstates, given in Eq. (13), or in the basis of mass eigenstates, according to Eq. (15).

This leaves open the choice of the free Lagrangian for the neutrino sector. In the following, we shall ignore the neutrino mass term in Eq. (11) and write a Lagrangian which is applicable in the high-energy limit. Following Ref. [17], we write it as

$$
\mathcal{L}=\sum_{\ell} \mathrm{i} \bar{\nu}_{\ell}^{(m)} \gamma^{\mu}\left(1-\gamma^{5}\right) \tilde{g}_{\mu \nu}\left(v_{\ell}\right) \partial^{\nu} \nu_{\ell}^{(m)},
$$

where

$$
\tilde{g}_{\mu \nu}\left(v_{\ell}\right)=\operatorname{diag}\left(1,-v_{\ell},-v_{\ell},-v_{\ell}\right)
$$

is a Lorentz-violating "metric" (pseudo-metric) describing the free propagation of the $\ell$ th mass eigenstate. We show in the following that the dispersion relation for the $\ell$ th mass eigenstate, implied by Eq. (16), is $E_{\ell}=|\vec{p}| v_{\ell}$.

Our aim here is to present a unified treatment of both LPCR and NPCR in the Lorentz-violating sector. The generalized interaction Lagrangian can be derived from the mass-basis interaction (15) upon considering the exchange of a $Z$ boson under kinematic conditions where the spatial momentum of the virtual exchange boson can be neglected. In Sec. II. 3 of [28], it has recently been shown that this is the case, for both tachyonic (Lorentz-conserving) and Lorentz-violating superluminal neutrinos, for surprisingly large incoming neutrino energies. Its range of applicability covers the entire kinematic regime from zero neutrino energy up to the "Big Bird" energy (Refs. [10,11]) of $2 \mathrm{PeV}$. We thus write the interaction Lagrangian as

$$
\begin{aligned}
\mathcal{L}_{\text {int }}= & f_{e} \frac{G_{F}}{2 \sqrt{2}} \bar{\nu}_{i}^{(m)} \gamma^{\lambda}\left(1-\gamma^{5}\right) \nu_{i}^{(m)} \\
& \times \tilde{g}_{\lambda \sigma}\left(v_{\text {int }}\right) \bar{\psi}_{f} \gamma^{\sigma}\left(c_{V}-c_{A} \gamma^{5}\right) \psi_{f} .
\end{aligned}
$$

When written in this form, the interaction Lagrangian subsumes both the form assumed in Ref. [26] (with $v_{\text {int }}=0$ ) and models I and II of Ref. [17] (with $v_{\text {int }}=0$ and $v_{\text {int }}=v_{i}$ respectively). On this occasion, we note that 
the difference between the results of Ref. [26] and model I of Ref. [17] arises due to the different treatment of the spin sum for the superluminal neutrino; see Eqs. (21) and (22) below. However, we can also keep $v_{\text {int }}$ as an additional free parameter of the model. As pointed out in Appendix B, gauge invariance with respect to a subgroup of the $S U(2)_{L} \times U(1)_{Y}$ electroweak gauge symmetry group is maintained for $v_{\text {int }}=v_{i} v_{f}$. This observation also explains the remark following Eq. (4) of Ref. [17], where the authors refer to a "gauge invariant" model. In Ref. [17], the authors assume that $v_{f}=1$. In Eq. (18), $\psi_{f}=e$ or $\nu_{f}^{(m)}$ is the fermion field for either the electron or a neutrino mass eigenstate, while

$$
f_{e}=\left\{\begin{array}{ll}
1, & \psi_{f}=\nu_{f}^{(m)} \\
2, & \psi_{f}=e
\end{array},\right.
$$

and (approximately)

$$
\left(c_{V}, c_{A}\right)=\left\{\begin{array}{ll}
(1,1) & \psi_{f}=\nu_{f}^{(m)} \\
\left(0,-\frac{1}{2}\right), & \psi_{f}=e
\end{array} .\right.
$$

In summary, we describe the decay processes depicted in Fig. 1 on the basis of a model which contains the following parameters:

(i) The parameter $\delta_{i}=v_{i}^{2}-1$ is the Lorentz-violating parameter for the initial, decaying particle state (comprising momenta $p_{1}$ and $p_{3}$ in Fig. 1).

(ii) The other Lorentz-violating parameter $\delta_{f}=v_{f}^{2}-1$ describes the produced pair (comprising momenta $p_{2}$ and $p_{4}$ in Fig. 1).

(iii) We have the Lorentz-violating pseudo-metric $\tilde{g}_{\mu \nu}\left(v_{\ell}\right)=\operatorname{diag}\left(1,-v_{\ell},-v_{\ell},-v_{\ell}\right)$ that enters the Dirac equation describing the neutrino mass eigenstates. (Here, $\ell$ can be the initial mass eigenstate $i$ or the final eigenstate $f$.) For an electron-positron final state, we may set $v_{\ell}=1$ at the end of the computation, according to the kinematic assumptions made.

(iv) A further model-dependent Lorentz-violating pseudo-metric $\tilde{g}_{\mu \nu}\left(v_{\text {int }}\right)$ enters the interaction Lagrangian given in Eq. (18) and has an additional free parameter,

$v_{\text {int }}$, where obviously $v_{\text {int }} \simeq 1$. In order to keep the interpretation of all parameters in our model similar, we set $v_{\text {int }}=\sqrt{1+\delta_{\text {int }}}$. As pointed out in Appendix B, gauge invariance with respect to a subgroup of $S U(2)_{L} \times U(1)_{Y}$ is maintained if we set $\delta_{\text {int }}=\delta_{i}+\delta_{f}$.

In order to keep the scope of the current investigation finite, we shall concentrate on the following cases:

(i) The Cohen-Glashow model for the LPCR process [26] has $\delta_{f}=0$ and replaces $\tilde{g}_{\mu \nu}\left(v_{\text {int }}\right) \rightarrow g_{\mu \nu}$ in the interaction Lagrangian, corresponding to $\delta_{\text {int }}=0$. Furthermore, the spin sum for a superluminal neutrino is assumed to take the following standard form:

$$
\sum_{s} \nu_{\ell, s} \otimes \bar{\nu}_{\ell, s}=p^{\mu} g_{\mu \nu} \gamma^{\nu}
$$

For the NPCR process, one uses these prescriptions but keeps $\delta_{f}$ as a free parameter.

(ii) The model I considered by Bezrukov and Lee [17] for the LPCR process also has $\delta_{f}=0$, and $\tilde{g}_{\mu \nu}\left(v_{\text {int }}\right)=g_{\lambda \sigma}$, corresponding to $\delta_{\text {int }}=0$; however the spin sum for a superluminal neutrino takes the form

$$
\sum_{s} \nu_{\ell, s} \otimes \bar{\nu}_{\ell, s}=p^{\mu} \tilde{g}_{\mu \nu}\left(v_{\ell}\right) \gamma^{\nu}
$$

as implied by the Dirac equation in Eq. (24). For the NPCR process, one again has to keep $\delta_{f}$ as a free parameter.

(iii) The model II considered by Bezrukov and Lee [17] for the LPCR process also has $\delta_{f}=0$, and $\tilde{g}_{\mu \nu}\left(v_{\text {int }}\right)=\tilde{g}_{\mu \nu}\left(v_{i}\right)$, which implies that $\delta_{\text {int }}=\delta_{i}$. Spin sums for superluminal neutrinos are evaluated as in Eq. (22). For the NPCR process, one again keeps $\delta_{f}$ as a free parameter. Models I and II of Bezrukov and Lee [17] can thus be unified on the basis of the parameter $\delta_{\text {int }}$, which assumes the value $\delta_{\text {int }}=0$ for model I and $\delta_{\text {int }}=\delta_{i}$ for model II. If one would like to maintain gauge invariance, within a subgroup of $S U(2) \times U(1)_{Y}$, and allow for a nonvanishing $\delta_{f}$, then one should replace $\delta_{\text {int }}=\delta_{i}+\delta_{f}$.

(iv) Expressed differently, one can interpolate between models I and II of Bezrukov and Lee [17] by considering $\delta_{\text {int }}$ to be an additional free parameter of the model.

All models considered use the pseudo-metric given in Eq. (17) for the description of initial and final states of the incoming and outgoing neutrinos.

\section{B. Formalism and models}

In order to study the LPCR and NPCR processes, $\nu_{i} \rightarrow$ $\nu_{i} \psi_{f} \bar{\psi}_{f}(\psi=e, \nu)$ in a unified way, we follow the framework laid out in Ref. [17], where the Lagrangian for a free superluminal neutrino field of flavor $i$ reads

$$
\mathcal{L}=\bar{\psi}_{i} \mathrm{i} \gamma^{\mu} \tilde{g}_{\mu \nu}\left(v_{i}\right) \partial^{\nu}\left(1-\gamma^{5}\right) \psi_{i},
$$

where the Lorentz-violating pseudo-metric $\tilde{g}_{\mu \nu}\left(v_{i}\right)$ has been defined in Eq. (17). As outlined above, we work with neutrino mass eigenstates and suppress the superscript $(m)$ in the following. Note that in comparison to Appendix A, we explicitly exhibit the left-handed chirality projection in 
Eq. (23), and we suppress the mass term. Crucially for what follows, the speed of the neutrino, $v_{i}=\sqrt{1+\delta_{i}}$, is allowed to depend on the flavor. The Lagrangian in Eq. (23) leads to the following superluminal Dirac equation:

$$
\gamma^{\mu} \tilde{g}_{\mu \nu}\left(v_{i}\right) p^{\nu} \psi_{i}=0
$$

by the usual variational equation. Upon multiplication from the right by the operator $\gamma^{\rho} \tilde{g}_{\rho \sigma}\left(v_{i}\right) p^{\nu} \psi_{i}=0$, the superluminal Dirac equation (24) implies that

$$
g^{\mu \nu} \tilde{g}_{\mu \alpha}\left(v_{i}\right) \tilde{g}_{\nu \beta}\left(v_{i}\right) p^{\alpha} p^{\beta} \psi_{i}=0,
$$

which in turn leads to the desired superluminal dispersion relation

$$
E^{2}-|\vec{k}| v_{i}^{2}=0,
$$

where we identify the components of the four-vector $p^{\nu}$ as $(E, \vec{k})$.

Before moving on to computing the decay rate, we pause briefly to reinterpret the above formulas in a form which is convenient for later calculations. The key observation is that by introducing the timelike vector

$$
t_{\mu}=(1,0,0,0),
$$

(assumed to take this form in the "laboratory" frame) we can rewrite the Lorentz-violating pseudo-metric $\tilde{g}_{\mu \nu}\left(v_{i}\right)$ as follows:

$$
\begin{aligned}
\tilde{g}_{\mu \nu}\left(v_{i}\right) & =v_{i} g_{\mu \nu}+\left(1-v_{i}\right) t_{\mu} t_{\nu} \\
& =\operatorname{diag}\left(1,-v_{i},-v_{i},-v_{i}\right) .
\end{aligned}
$$

A remark is in order. In Ref. [31], a potentially necessary field redefinition has been discussed, in order to ensure that the fields are canonically normalized in a Lorentz-violating scenario. However, we note that, for the pseudo-metric (17), the field redefinition transformation $A$ outlined in Eqs. (4), (10) and (11) of Ref. [31] amounts to the unity transformation; hence no further corrections are incurred. This can be seen as follows. First, we notice that the pseudo-metric $\tilde{g}_{\mu \nu}\left(v_{i}\right)$ has the properties $\tilde{g}_{00}\left(v_{i}\right)=1$ and $\tilde{g}_{0 \nu}\left(v_{i}\right)=\delta^{0 \nu}$, and hence, no extra time derivative pieces are introduced in the modified action or modified Dirac equation.

In the notation of Ref. [31], our model corresponds to the parameter choice

$$
c_{i}^{\mu \nu}=\left(v_{i}-1\right) \operatorname{diag}(0,-1,-1,-1) .
$$

The spinor redefinition of Ref. [31], which otherwise eliminates the extra time derivatives, amounts to the transformation

$$
\psi=A \chi, \quad A=1-\frac{1}{2} c_{\mu 0} \gamma^{0} \gamma^{\mu},
$$

but in our model $c_{\mu 0}=0$, so that we simply have $A=1$; i.e., no spinor redefinition is needed.

The superluminal Dirac equation in Eq. (24) takes the form

$$
\begin{aligned}
\gamma^{\mu}\left[v_{i} g_{\mu \nu}+\left(1-v_{i}\right) t_{\mu} t_{\nu}\right] p^{\nu} \psi_{i} & =\left[v_{i} \not \supset+\left(1-v_{i}\right)(p \cdot t) f\right] \psi_{i} \\
& =0,
\end{aligned}
$$

where the slashed notation always has its usual meaning of $\not l=\gamma^{\mu} g_{\mu \nu} a^{\nu}$ for any four-vector $a$, with $g_{\mu \nu}$ the usual pseudo-metric. Furthermore, the spin sum in Eq. (22) can be written as

$$
\sum_{s} \nu_{i, s} \otimes \bar{\nu}_{i, s}=v_{i} \not p+\left(1-v_{i}\right)(p \cdot t) t .
$$

Clearly, this differs from the standard spin sum of Eq. (21), $\sum_{s} \nu_{i, s} \otimes \bar{\nu}_{i, s}=\not p$, by terms that are first order in $\delta_{i}$, as already pointed out in Ref. [17].

With the effective interaction Lagrangian given in Eq. (18), we are now ready to consider the emission of a fermion pair from a superluminal neutrino in the most general setup with fermion flavor-dependent speeds as well as a generic $v_{\text {int }}$.

\section{CALCULATION OF EMISSION RATES}

\section{A. Matrix elements}

Let us consider the process

$$
\nu_{i}\left(p_{1}\right) \rightarrow \nu_{i}\left(p_{3}\right)+\bar{\psi}_{f}\left(p_{2}\right)+\psi_{f}\left(p_{4}\right),
$$

where we have indicated in the parentheses the momentum assignment as in Fig. 1. As discussed above, we work in the mass basis for the neutrinos, and we can specify the type of fermion $\psi$ at the very end of the calculation.

The transition matrix element for the process in Eq. (33) can be computed from the effective interaction Lagrangian given in Eq. (18):

$$
\begin{aligned}
\mathcal{M}= & f_{e} \frac{G_{F}}{2 \sqrt{2}}\left[\bar{u}_{i}\left(p_{3}\right) \gamma^{\lambda}\left(1-\gamma^{5}\right) u_{i}\left(p_{1}\right)\right] \tilde{g}_{\lambda \sigma}\left(v_{\text {int }}\right) \\
& \times\left[\bar{u}_{f}\left(p_{4}\right)\left(c_{V} \gamma^{\sigma}-c_{A} \gamma^{\sigma} \gamma^{5}\right) v_{f}\left(p_{2}\right)\right] .
\end{aligned}
$$

Summation over the spins of the final state particles and averaging over those of the initial ones can be performed using standard trace technology. However, one must remember that except for the Cohen-Glashow model, the spin sums for superluminal particles should be written as in (32). Allowing for $n_{s}$ spin states of the neutrino $\left(n_{s}=2\right.$ in Ref. [26] but $n_{s}=1$ in Ref. [17]), we find 


$$
\begin{aligned}
\frac{1}{n_{s}} \sum_{\text {spins }}|\mathcal{M}|^{2}= & \frac{1}{n_{s}} f_{e}^{2} \frac{G_{F}^{2}}{8} \operatorname{Tr}\left[\left(v_{i} \not \not_{3}+\left(1-v_{i}\right)\left(p_{3} \cdot t\right) t\right) \gamma^{\lambda}\left(1-\gamma^{5}\right)\left(v_{i} \not \not_{1}+\left(1-v_{i}\right)\left(p_{1} \cdot t\right) \not\right) \gamma^{\sigma}\left(1-\gamma^{5}\right)\right] \\
& \times\left[v_{\text {int }} g_{\lambda \rho}+\left(1-v_{\text {int }}\right) t_{\lambda} t_{\rho}\right]\left[v_{\text {int }} g_{\sigma \tau}+\left(1-v_{\text {int }}\right) t_{\sigma} t_{\tau}\right] \operatorname{Tr}\left[\left(v_{f} \not{ }_{4}+\left(1-v_{f}\right)\left(p_{4} \cdot t\right) t\right)\left(c_{V} \gamma^{\rho}-c_{A} \gamma^{\rho} \gamma^{5}\right)\right. \\
& \left.\times\left(v_{f} \not \not_{2}+\left(1-v_{f}\right)\left(p_{2} \cdot t\right) f\right)\left(c_{V} \gamma^{\tau}-c_{A} \gamma^{\tau} \gamma^{5}\right)\right] .
\end{aligned}
$$

Let us remark here, for absolute clarification, that the use of the convention $n_{s}=1$ in Ref. [17] of course does not imply that neutrinos are treated as scalar particles by Bezrukov and Lee, but only means that the authors assumed that all oncoming particles have left-handed helicity. If we were to use $n_{s}=2$ in all calculations reported below for the models of Bezrukov and Lee [17], then the corresponding results would have to be divided by a factor 2 .
The computation of traces and contractions is straightforward, although cumbersome. The final result is somewhat long and will not be exhibited here, but the general structure is the following. The squared matrix element can be written as a linear combination of ten different kinematic structures, all of mass dimension four:

$$
\begin{aligned}
\frac{1}{n_{s} \sum_{\text {spins }}|\mathcal{M}|^{2}=} & \frac{1}{n_{s}} f_{e}^{2} \frac{G_{F}^{2}}{8}\left[c_{1}\left(p_{1} \cdot p_{2}\right)\left(p_{3} \cdot p_{4}\right)+c_{2}\left(p_{1} \cdot p_{3}\right)\left(p_{2} \cdot p_{4}\right)+c_{3}\left(p_{1} \cdot p_{4}\right)\left(p_{2} \cdot p_{3}\right)\right. \\
& +c_{4}\left(p_{1} \cdot p_{2}\right)\left(p_{3} \cdot t\right)\left(p_{4} \cdot t\right)+c_{5}\left(p_{1} \cdot p_{3}\right)\left(p_{2} \cdot t\right)\left(p_{4} \cdot t\right)+c_{6}\left(p_{1} \cdot p_{4}\right)\left(p_{2} \cdot t\right)\left(p_{3} \cdot t\right) \\
& +c_{7}\left(p_{2} \cdot p_{3}\right)\left(p_{1} \cdot t\right)\left(p_{4} \cdot t\right)+c_{8}\left(p_{2} \cdot p_{4}\right)\left(p_{1} \cdot t\right)\left(p_{3} \cdot t\right)+c_{9}\left(p_{3} \cdot p_{4}\right)\left(p_{1} \cdot t\right)\left(p_{2} \cdot t\right) \\
& \left.+c_{10}\left(p_{1} \cdot t\right)\left(p_{2} \cdot t\right)\left(p_{3} \cdot t\right)\left(p_{4} \cdot t\right)\right] .
\end{aligned}
$$

Here the $c_{i}(i=1, \ldots, 10)$ are numerical coefficients that depend on $v_{i}, v_{f}, v_{\text {int }}$ as well as $c_{A}, c_{V}$ and whether Eq. (21) or Eq. (22) is used to evaluate the spin sum for superluminal particles.

\section{B. Phase-space integration}

Now we are ready to compute the fermion emission rate, which essentially amounts to integrating the squared matrix element in Eq. (36) over the available phase space:

$$
\Gamma=\frac{1}{2 E_{1}} \int \mathrm{d} \phi_{3}\left(p_{2}, p_{3}, p_{4} ; p_{1}\right) \frac{1}{n_{s}} \sum_{\text {spins }}|\mathcal{M}|^{2} .
$$

A remark is in order. Namely, in Ref. [31], small deviations of the formulas for the calculation of cross sections in Lorentz-violating theories, from those in Lorentz-invariant theories, have been derived. A closer inspection of Ref. [31] reveals that the correction terms to the flux factors are of relative order of the $\delta$ parameters of the oncoming particles (assuming that the deviations from the speed of light are small, $\delta \ll 1$ ). The additional corrections would thus modify the results given in Sec. IV A at higher order in the expansion parameters $\delta$, which we consider the terms to be numerically small. In addition, a closer inspection reveals that the tiny modifications necessary for the cross sections in Lorentz-violating theories can be traced to a flux factor which involves the relative velocity of the in- coming particles, as shown in Ref. [31]. However, the formula for the decay rate does not involve a flux factor, and hence, Eq. (37) does not require further modifications. The same approach has been taken in Refs. [17,26].

We evaluate the integral by first using the splitting relation [32] (in the "laboratory" frame) to write

$$
\begin{aligned}
\Gamma= & \frac{1}{2 E_{1}} \int_{M_{\min }^{2}}^{M_{\max }^{2}} \frac{\mathrm{d} M^{2}}{2 \pi} \mathrm{d} \phi_{2}\left(p_{3}, p_{24} ; p_{1}\right) \mathrm{d} \phi_{2}\left(p_{2}, p_{4} ; p_{24}\right) \\
& \times \frac{1}{n_{s}} \sum_{\text {spins }}|\mathcal{M}|^{2} .
\end{aligned}
$$

The product of the two factors, $\mathrm{d} \phi_{2}\left(p_{3}, p_{24} ; p_{1}\right)$ times $\mathrm{d} \phi_{2}\left(p_{2}, p_{4} ; p_{24}\right)$, displays the kinematics of the process, in two steps, $p_{1} \rightarrow p_{3}+p_{24}$ and $p_{24} \rightarrow p_{2}+p_{4}$. Although the splitting relation is well known [32] and has been used by us in the analysis of the three-jet phase space in quantum chromodynamics in next-to-next-to-leading order [see Eq. (2.9) of Ref. [33] and Eq. (2.12) of Ref. [34]], we give a quick derivation here to emphasize the fact that it holds without change also for superluminal momenta. We start with the definition of the three-particle phase space for the final state, $\mathrm{d} \phi_{3}$,

$$
\begin{aligned}
\mathrm{d} \phi_{3}( & \left.p_{2}, p_{3}, p_{4} ; p_{1}\right) \\
= & \frac{\mathrm{d}^{4} p_{2}}{(2 \pi)^{3}} \delta_{+}\left(p_{2}^{2}-\delta_{f} k_{2}^{2}\right) \\
& \times \frac{\mathrm{d}^{4} p_{3}}{(2 \pi)^{3}} \delta_{+}\left(p_{3}^{2}-\delta_{i} k_{3}^{2}\right) \frac{\mathrm{d}^{4} p_{4}}{(2 \pi)^{3}} \delta_{+}\left(p_{4}^{2}-\delta_{f} k_{4}^{2}\right) \\
& \times(2 \pi)^{4} \delta^{(4)}\left(p_{1}-p_{2}-p_{3}-p_{4}\right),
\end{aligned}
$$


where we recall the definition of the components of the four-vector $p^{\nu}$ as $p^{\nu}=(E, \vec{k})$, from Eq. (26), and note that

$$
\begin{aligned}
g^{\mu \nu} \tilde{g}_{\mu \alpha} \tilde{g}_{\mu \beta} p^{\alpha} p^{\beta} & =E^{2}-v^{2} \vec{k}^{2}=g_{\mu \nu} p^{\mu} p^{\nu}-\left(v^{2}-1\right) \vec{k}^{2} \\
& =p^{2}-\delta k^{2} .
\end{aligned}
$$

Furthermore,

$$
\delta_{+}\left(p^{2}-\delta k^{2}\right)=\delta_{+}\left(E^{2}-v^{2} k^{2}\right)=\frac{1}{2 E} \delta(E-v|k|)
$$

identifies a Dirac- $\delta$ which is nonzero only for positive values of $E=p^{0}$. We now insert a factor of 1, written in the following form:

$$
1=\int \mathrm{d}^{4} p_{24} \delta^{(4)}\left(p_{24}-p_{2}-p_{4}\right) \int \mathrm{d} M^{2} \delta_{+}\left(p_{24}^{2}-M^{2}\right) .
$$

The second delta function implies that $p_{24}$ is a generic massive momentum. This is appropriate, since in general the sum of two superluminal momenta will not satisfy the superluminal dispersion relation in the "laboratory" frame. Thus we find

$$
\begin{aligned}
\mathrm{d} \phi_{3}\left(p_{2}, p_{3}, p_{4} ; p_{1}\right)= & \int \frac{\mathrm{d} M^{2}}{2 \pi} \frac{\mathrm{d}^{4} p_{3}}{(2 \pi)^{3}} \delta_{+}\left(p_{3}^{2}-\delta_{i} k_{3}^{2}\right) \frac{\mathrm{d}^{4} p_{24}}{(2 \pi)^{3}} \delta_{+}\left(p_{24}^{2}-M^{2}\right)(2 \pi)^{4} \delta^{(4)}\left(p_{1}-p_{3}-p_{24}\right) \\
& \times \frac{\mathrm{d}^{4} p_{2}}{(2 \pi)^{3}} \delta_{+}\left(p_{2}^{2}-\delta_{f} k_{2}^{2}\right) \frac{\mathrm{d}^{4} p_{4}}{(2 \pi)^{3}} \delta_{+}\left(p_{4}^{2}-\delta_{f} k_{4}^{2}\right)(2 \pi)^{4} \delta^{(4)}\left(p_{24}-p_{2}-p_{4}\right) \\
= & \int \frac{\mathrm{d} M^{2}}{2 \pi} \mathrm{d} \phi_{2}\left(p_{3}, p_{24} ; p_{1}\right) \mathrm{d} \phi_{2}\left(p_{2}, p_{4} ; p_{24}\right) ;
\end{aligned}
$$

i.e., the result is formally identical to the usual case with no superluminal particles.

We must now work out the limits of integration of the $M^{2}$ integral. To do this, let us recall that by momentum conservation $\left(p_{1}^{\mu}=p_{2}^{\mu}+p_{3}^{\mu}+p_{4}^{\mu}\right)$ we have

$$
\begin{aligned}
& p_{24}^{\mu}=p_{2}^{\mu}+p_{4}^{\mu}=p_{1}^{\mu}-p_{3}^{\mu}, \\
& M^{2}=p_{24}^{2}=\left(p_{1}-p_{3}\right)^{2} .
\end{aligned}
$$

However, using the superluminal dispersion relation, we find

$$
\begin{aligned}
\left(p_{1}-p_{3}\right)^{2}= & p_{1}^{2}+p_{3}^{2}-2 p_{1} \cdot p_{3} \\
= & \delta_{i}\left|\vec{p}_{1}\right|^{2}+\delta_{i}\left|\vec{p}_{3}\right|^{2}-2\left(1+\delta_{i}\right)\left|\vec{p}_{1}\right|\left|\vec{p}_{3}\right| \\
& +2\left|\vec{p}_{1}\right|\left|\vec{p}_{3}\right| \cos \theta_{13},
\end{aligned}
$$

where $\theta_{13}$ is the angle between the three-momenta $\vec{p}_{1}$ and $\vec{p}_{3}$. Clearly, the expression above attains its maximum when $\cos \theta_{13}=1$, so

$$
M_{\max }^{2}=\delta_{i}\left(\left|\vec{p}_{1}\right|-\left|\vec{p}_{3}\right|\right)^{2} .
$$

On the other hand, we have also

$$
\begin{aligned}
M^{2}= & \left(p_{2}+p_{4}\right)^{2}=p_{2}^{2}+p_{4}^{2}+2 p_{2} \cdot p_{4} \\
= & \delta_{f}\left|\vec{p}_{2}\right|^{2}+\delta_{f}\left|\vec{p}_{4}\right|^{2}+2\left(1+\delta_{f}\right)\left|\vec{p}_{2}\right|\left|\vec{p}_{4}\right| \\
& -2\left|\vec{p}_{2}\right|\left|\vec{p}_{4}\right| \cos \theta_{24},
\end{aligned}
$$

where $\theta_{24}$ is now the angle between the three-momenta $\vec{p}_{2}$ and $\vec{p}_{4}$. Obviously, the expression above is minimal when $\cos \theta_{24}=1$, so

$$
M_{\min }^{2}=\delta_{f}\left(\left|\vec{p}_{2}\right|+\left|\vec{p}_{4}\right|\right)^{2} .
$$

However, energy conservation in the laboratory frame simply reads

$\sqrt{1+\delta_{i}}\left|\vec{p}_{1}\right|=\sqrt{1+\delta_{f}}\left|\vec{p}_{2}\right|+\sqrt{1+\delta_{i}}\left|\vec{p}_{3}\right|+\sqrt{1+\delta_{f}}\left|\vec{p}_{4}\right|$,

implying

$$
\left|\vec{p}_{2}\right|+\left|\vec{p}_{4}\right|=\frac{\sqrt{1+\delta_{i}}}{\sqrt{1+\delta_{f}}}\left(\left|\vec{p}_{1}\right|-\left|\vec{p}_{3}\right|\right)
$$

and hence finally

$$
\begin{aligned}
& M_{\max }^{2}=\delta_{i}\left(\left|\vec{p}_{1}\right|-\left|\vec{p}_{3}\right|\right)^{2}, \\
& M_{\min }^{2}=\delta_{f} \frac{1+\delta_{i}}{1+\delta_{f}}\left(\left|\vec{p}_{1}\right|-\left|\vec{p}_{3}\right|\right)^{2} .
\end{aligned}
$$

In particular, for $\delta_{i}=\delta_{f}$ the limits coincide and hence there is no phase space for the decay. (For $\delta_{i}<\delta_{f}$ the maximum allowed value of $M^{2}$ would be lower than the minimum allowed value.)

The utility of the splitting relation lies in the fact that we can first perform the integration of the squared matrix element over the momenta of the two outgoing "slow" 
particles (where "slow" refers to a potentially still superluminal particle with $\delta_{f}<\delta_{i}$ ). These are $p_{2}$ and $p_{4}$. To do this, we must evaluate the tensor integral (for $p_{2}$ and $p_{4}$ both superluminal)

$$
J^{\mu \nu}\left(p_{24}\right)=\int \mathrm{d} \phi_{2}\left(p_{2}, p_{4} ; p_{24}\right) p_{2}^{\mu} p_{4}^{\nu}
$$

This can be computed using the following explicit parametrization of the two-particle phase space $\mathrm{d} \phi_{2}\left(p_{2}, p_{4} ; p_{24}\right)$, valid when both $p_{2}$ and $p_{4}$ are superluminal with speed $v_{f}$ :

$$
\begin{aligned}
& \int \mathrm{d} \phi_{2}\left(p_{2}, p_{4} ; p_{24}\right) \\
& =\frac{1}{8 \pi} \int_{\left|\vec{p}_{2}\right|_{\min }}^{\left|\vec{p}_{2}\right|_{\max }} \frac{\mathrm{d}\left|\vec{p}_{2}\right| \mathrm{d}(\cos \theta)}{\left(1+\delta_{f}\right)^{3 / 2}\left|\vec{p}_{24}\right|} \\
& \times \delta\left[\cos \theta-\frac{2 \sqrt{1+\delta_{f}} E_{24}\left|\vec{p}_{2}\right|+\delta_{f}\left|\vec{p}_{24}\right|^{2}-M^{2}}{2\left(1+\delta_{f}\right)\left|\vec{p}_{24}\right|\left|\vec{p}_{2}\right|}\right],
\end{aligned}
$$

where the limits of integration read

$$
\begin{aligned}
& \left|\vec{p}_{2}\right|_{\max }=\frac{E_{24}+\sqrt{1+\delta_{f}}\left|\vec{p}_{24}\right|}{2 \sqrt{1+\delta_{f}}}, \\
& \left|\vec{p}_{2}\right|_{\text {min }}=\frac{E_{24}-\sqrt{1+\delta_{f}}\left|\vec{p}_{24}\right|}{2 \sqrt{1+\delta_{f}}} .
\end{aligned}
$$

Then the tensor integral can be computed (e.g., component by component) and we find

$$
\begin{aligned}
J^{\mu \nu}\left(p_{24}\right)= & \frac{1}{12}\left[\frac{E_{24}^{2}-\left(1+\delta_{f}\right)\left|\vec{p}_{24}\right|^{2}}{1+\delta_{f}} g^{\mu \nu}+2 p_{24}^{\mu} p_{24}^{\nu}\right. \\
& \left.+\delta_{f} \frac{E_{24}^{2}-\left(1+\delta_{f}\right)\left|\vec{p}_{24}\right|^{2}}{1+\delta_{f}} t^{\mu} t^{\nu}\right] V_{2}^{2 \mathrm{SL}}\left(\delta_{f}\right),
\end{aligned}
$$

where $V_{2}^{2 \mathrm{SL}}\left(\delta_{f}\right)$ is the volume of the two-body phase space when both particles are superluminal and have a speed of $v_{f}$,

$$
V_{2}^{2 \mathrm{SL}}\left(\delta_{f}\right)=\frac{1}{8 \pi\left(1+\delta_{f}\right)^{3 / 2}}
$$

Substituting this, we are left with the integral over the two-body phase space $\mathrm{d} \phi_{2}\left(p_{3}, p_{24} ; p_{1}\right)$ and over $\mathrm{d} M^{2}$. The integration is essentially straightforward; however, care must be taken to properly identify the limits of integration in all variables. In particular, the phase space for one superluminal momentum with speed $v_{i}$ and one massive momentum with mass $M^{2}$ can be written in the following explicit form:

$$
\begin{aligned}
& \int \mathrm{d} \phi_{2}\left(p_{3}, p_{24} ; p_{1}\right) \\
& =\frac{1}{8 \pi} \int_{\left|\vec{p}_{3}\right|_{\min }}^{\left|\vec{p}_{3}\right|_{\max } \frac{\mathrm{d}\left|\vec{p}_{3}\right| \mathrm{d}(\cos \theta)}{\sqrt{1+\delta_{i} \mid}\left|\vec{p}_{1}\right|}} \\
& \quad \times \delta\left[\cos \theta-\frac{2\left(1+\delta_{i}\right)\left|\vec{p}_{1}\right|\left|\vec{p}_{3}\right|+M^{2}-\delta_{i}\left|\vec{p}_{1}\right|^{2}-\delta_{i}\left|\vec{p}_{3}\right|^{2}}{2\left|\vec{p}_{1}\right|\left|\vec{p}_{3}\right|}\right],
\end{aligned}
$$

where $\theta$ is the angle between the incoming and outgoing three-momentum of the "fast" superluminal neutrino. The limits of integration are

$$
\begin{aligned}
& \left|\vec{p}_{3}\right|_{\text {max }}=\left|\vec{p}_{1}\right|-\frac{M}{\sqrt{\delta_{i}}}, \\
& \left|\vec{p}_{3}\right|_{\text {min }}=\frac{\left(2+\delta_{i}\right)\left|\vec{p}_{1}\right|-\sqrt{4\left(1+\delta_{i}\right)\left|\vec{p}_{1}\right|^{2}+\delta_{i} M^{2}}}{\delta_{i}} .
\end{aligned}
$$

However, phase space is also constrained by the limits of the $\mathrm{d} M^{2}$ integration, given in Eqs. (51a) and (51b). As remarked earlier, if $\delta_{i}=\delta_{f}$, then $M_{\max }^{2}$ and $M_{\min }^{2}$ coincide and there is no phase space for the decay. A careful but straightforward analysis establishes that the true region of integration corresponds to

$$
\left|\vec{p}_{3}\right|_{\text {min }} \leq\left|\vec{p}_{3}\right| \leq\left|\vec{p}_{3}\right|_{\max }
$$

only for

$$
M_{\mathrm{cut}}^{2} \leq M^{2} \leq \delta_{i}\left|\vec{p}_{1}\right|^{2}
$$

while on the other hand

$$
\left|\vec{p}_{1}\right|-\sqrt{\frac{1+\delta_{f}}{1+\delta_{i}}} \frac{M}{\sqrt{\delta_{f}}} \leq\left|\vec{p}_{3}\right| \leq\left|\vec{p}_{3}\right|_{\max }
$$

if

$$
0 \leq M^{2} \leq M_{\mathrm{cut}}^{2}
$$

where

$$
M_{\mathrm{cut}}^{2}=4 \delta_{f}\left(1+\delta_{i}\right) \frac{\left(\sqrt{1+\delta_{i}}-\sqrt{1+\delta_{f}}\right)^{2}}{\left(\delta_{i}-\delta_{f}\right)^{2}}\left|\vec{p}_{1}\right|^{2} .
$$

Thus, the integrals can be performed most easily by splitting the $\mathrm{d} M^{2}$ integration at $M_{\text {cut }}^{2}$. The physical region in the $\left(M^{2},\left|\vec{p}_{3}\right|\right)$ plane is shown in Fig. 2.

Before presenting our results, let us comment briefly on the computation of the differential energy loss, given by [28] 


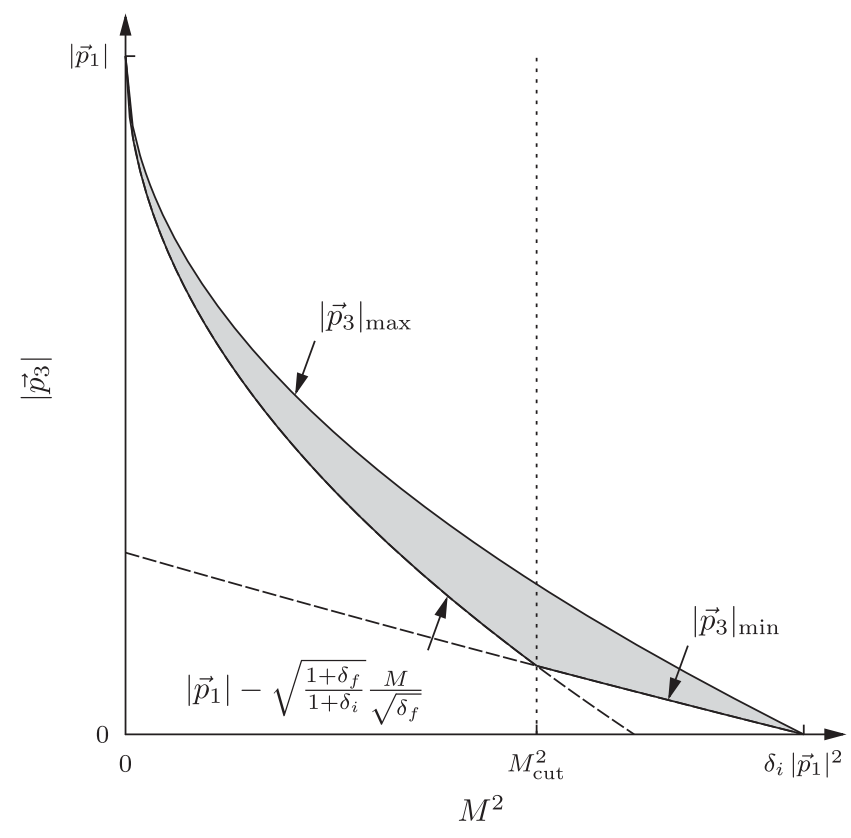

FIG. 2. The physical region (shaded) in the $\left(M^{2},\left|\vec{p}_{3}\right|\right)$ plane for "slow" superluminal neutrino pair emission from a "fast" superluminal neutrino. $\left|\vec{p}_{3}\right|_{\text {max }}$ and $\left|\vec{p}_{3}\right|_{\text {min }}$ are defined in Eq. (58), while $M_{\text {cut }}^{2}$ is given in Eq. (63). The variable $M^{2}$ is introduced in Eq. (42) [see also Eq. (51)].

$$
\frac{\mathrm{d} E_{1}}{\mathrm{~d} x}=-\int \mathrm{d} E_{3}^{\prime}\left(E_{1}-E_{3}^{\prime}\right) \frac{\mathrm{d} \Gamma}{\mathrm{d} E_{3}^{\prime}}
$$

This formula first and foremost applies to the energy loss per time, which translates into an energy loss per traveled distance for particle velocities near the speed of light $(c=1$ in our conventions). The differential decay rate is then simply

$$
\frac{\mathrm{d} \Gamma}{\mathrm{d} E_{3}^{\prime}}=\frac{1}{2 E_{1}} \int \phi_{3}\left(p_{2}, p_{3}, p_{4} ; p_{1}\right) \frac{1}{2} \sum_{\text {spins }}|\mathcal{M}|^{2} \delta\left(E_{3}-E_{3}^{\prime}\right) .
$$

When evaluating this expression, we need to keep in mind that in our parametrization of phase space [see Eq. (57)], $E_{3}$ is assumed to be expressed as a function of $\left|\vec{p}_{3}\right|$, i.e., $E_{3}=\sqrt{1+\delta_{i}}\left|\vec{p}_{3}\right|$. Hence we must replace $E_{3}$ in the above equation by $\sqrt{1+\delta_{i}}\left|\vec{p}_{3}\right|$. In particular, the Dirac delta function in the above equation becomes $\delta\left(\sqrt{1+\delta_{i}}\left|\vec{p}_{3}\right|-E_{3}^{\prime}\right)$. We can then perform the $E_{3}^{\prime}$ integration in (64) with this delta function first, so

$$
\begin{aligned}
\frac{\mathrm{d} E_{1}}{\mathrm{~d} x} & =-\int \mathrm{d} E_{3}^{\prime}\left(E_{1}-E_{3}^{\prime}\right) \frac{\mathrm{d} \Gamma}{\mathrm{d} E_{3}^{\prime}} \\
& =-\int \mathrm{d} E_{3}^{\prime}\left(E_{1}-E_{3}^{\prime}\right) \frac{1}{2 E_{1}} \int \mathrm{d} \phi_{3}\left(p_{2}, p_{3}, p_{4} ; p_{1}\right) \frac{1}{2} \sum_{\text {spins }}|\mathcal{M}|^{2} \delta\left(E_{3}-E_{3}^{\prime}\right) \\
& =-\frac{1}{2 E_{1}} \int \mathrm{d} \phi_{3}\left(p_{2}, p_{3}, p_{4} ; p_{1}\right) \sqrt{1+\delta_{i}}\left(\left|\vec{p}_{1}\right|-\left|\vec{p}_{3}\right|\right) \frac{1}{2} \sum_{\text {spins }}|\mathcal{M}|^{2}
\end{aligned}
$$

where we have used $E_{1}=\sqrt{1+\delta_{i}}\left|\vec{p}_{1}\right|$ and $E_{3}=\sqrt{1+\delta_{i}}\left|\vec{p}_{3}\right|$. We can perform the integration over the phase space exactly as before; in fact, the calculation can be mapped onto the one for the decay rate by simply replacing $|\mathcal{M}|^{2} \rightarrow-\sqrt{1+\delta_{i}}\left(\left|\vec{p}_{1}\right|-\left|\vec{p}_{3}\right|\right)|\mathcal{M}|^{2}$.

\section{DECAY AND ENERGY LOSS RATES}

\section{A. Analytic results}

The complete results for the decay rate and differential energy loss in full generality are quite cumbersome. Hence, inspired by the approach of Ref. [26], we present here only the leading order results in the small quantities $\delta$, but keep all terms of third order in the $\delta$ parameters. More precisely, we assume that $\delta_{i} \sim \delta_{f} \sim \delta_{\text {int }}$ are all of the same order of magnitude and perform an expansion to the first nonvanishing order.

First, we present a general result, namely, for the total rate for the process $\nu_{i} \rightarrow \nu_{i} \psi_{f} \bar{\psi}_{f}$ (where $\psi_{f}$ and $\bar{\psi}_{f}$ can be any of the discussed outgoing fermions and antifermions, i.e., an electron-positron or a neutrino-antineutrino pair). This general result can be written in the following form, for a Lorentz-violating parameter $\delta_{i}$ of the incoming neutrino, and $\delta_{f}$ for the outgoing particle-antiparticle pair:

$$
\begin{aligned}
\Gamma_{\nu_{i} \rightarrow \nu_{i} \psi_{f} \bar{\psi}_{f}}= & \frac{G_{F}^{2} k_{1}^{5}}{192 \pi^{3}} f_{e}^{2} \frac{c_{V}^{2}+c_{A}^{2}}{420 n_{s}}\left(\delta_{i}-\delta_{f}\right) \\
& \times\left[\left(60-43 \sigma_{i}\right)\left(\delta_{i}-\delta_{f}\right)^{2}\right. \\
& +2\left(50-32 \sigma_{i}-25 \sigma_{f}+7 \sigma_{i} \sigma_{f}\right)\left(\delta_{i}-\delta_{f}\right) \delta_{f} \\
& \left.+7\left(4-3 \sigma_{i}-3 \sigma_{f}+2 \sigma_{i} \sigma_{f}\right) \delta_{f}^{2}+7 \delta_{\mathrm{int}}^{2}\right],
\end{aligned}
$$

where we notice the parameter $n_{s}$ for the number of active spin states in the denominator. Furthermore, $\sigma_{i}$ and $\sigma_{f}$ are 0 or 1 depending on whether the Cohen-Glashow (CG) [see Ref. [26] and Eq. (21)] or the Bezrukov-Lee (BL) [see Ref. [17] and Eq. (32)] prescription is adopted for the sum over spins of the initial and final particles: 


$$
\sigma_{i}= \begin{cases}0, & \mathrm{CG} \text { spin sum for } \nu_{i} \\ 1, & \mathrm{BL} \text { spin sum for } \nu_{i}\end{cases}
$$

and

$$
\sigma_{f}= \begin{cases}0, & \text { CG spin sum for } \psi_{f}, \\ 1, & \text { BL spin sum for } \psi_{f}\end{cases}
$$

Furthermore, $\delta_{\text {int }}=v_{\text {int }}^{2}-1$ is the Lorentz-violating parameter for the pseudo-metric used in the interaction Lagrangian (18), and $n_{s}$ is the number of available spin states assumed in a particular model. For the differential energy loss, we find

$$
\begin{aligned}
\frac{\mathrm{d} E_{\nu_{i} \rightarrow \nu_{i} \psi_{f} \bar{\psi}_{f}}}{\mathrm{~d} x}= & -\frac{G_{F}^{2} k_{1}^{6}}{192 \pi^{3}} f_{e}^{2} \frac{c_{V}^{2}+c_{A}^{2}}{672 n_{s}}\left(\delta_{i}-\delta_{f}\right) \\
& \times\left[\left(75-53 \sigma_{i}\right)\left(\delta_{i}-\delta_{f}\right)^{2}\right. \\
& +\left(122-77 \sigma_{i}-61 \sigma_{f}+16 \sigma_{i} \sigma_{f}\right)\left(\delta_{i}-\delta_{f}\right) \delta_{f} \\
& \left.+8\left(4-3 \sigma_{i}-3 \sigma_{f}+2 \sigma_{i} \sigma_{f}\right) \delta_{f}^{2}+8 \delta_{\mathrm{int}}^{2}\right]
\end{aligned}
$$

with $\sigma_{i}$ and $\sigma_{f}$ as above. Using the formulas in Eqs. (67) and (70), we can rederive the results of Refs. $[17,26]$ by an appropriate choice of the various parameters, as discussed in Sec. II. It is interesting to observe that in Eq. (70), terms proportional to $c_{V} c_{A}$ vanish. This conclusion can be supported by a detailed analysis of the Dirac algebra of the transition currents and phase-space integrals: Namely, conceivable contributions proportional to $c_{V} c_{A}$ would be multiplied by an antisymmetric Dirac structure (in the indices of the outgoing pair), multiplied by a symmetric phase-space integral, and hence, they vanish.

We now proceed to the indication of the results for the superluminal models discussed here. Because of a certain multitude of models discussed here, let us anticipate, for the convenience of the reader, the following conventions:

(i) The $a$ and $a^{\prime}$ coefficients given below in Eq. (74) refer to the vacuum pair emission, or LPCR, with $a$ entering the formula for the decay rate, while $a^{\prime}$ enters the formula for the energy loss rate.

(ii) The $b$ and $b^{\prime}$ coefficients given below in Eqs. (82) and (83) refer to the neutrino-splitting or NPCR, with $b$ entering the formula for the decay rate, while $b^{\prime}$ enters the formula for the energy loss rate.

(iii) Coefficients with a subscript CG refer to the CohenGlashow model [26], which assumes the spin sum (21) and has $n_{s}=2$.

(iv) Coefficients with a subscript BL refer to the BezrukovLee model [17], which assumes the spin sum (22) and has $n_{s}=1$.

(v) The parameter $\delta_{\text {int }}$ enters the effective interaction Lagrangian (18). According to the discussion in Appendixes A and B (see also Ref. [27]), a restricted gauge structure (with a reduced symmetry group) of the electroweak interaction is preserved for $\delta_{\text {int }}=\delta_{i}+\delta_{e} \quad$ (LPCR process) and $\delta_{\text {int }}=\delta_{i}+\delta_{f}$ (NPCR process). Here, the parameters $\delta_{i}, \delta_{e}$ and $\delta_{f}$ are measured with respect to the speed of light (see also Ref. [35]).

Starting with the case $\psi_{f}=e$ (LPCR process), we recover the models of Cohen and Glashow [using the standard spin sum of Eq. (21), i.e., $\sigma_{i}=\sigma_{f}=0$ ] of Ref. [26] and of Bezrukov and Lee [using the superluminal spin sum of Eq. (22), i.e., $\sigma_{i}=\sigma_{f}=1$ ] of Ref. [17]. We obtain for $\delta_{f}=\delta_{e}=0$,

$$
\begin{gathered}
\Gamma_{\nu_{i} \rightarrow \nu_{i} e^{-} e^{+}}=a \frac{G_{F}^{2}}{192 \pi^{3}} k_{1}^{5}, \\
\frac{\mathrm{d} E_{\nu_{i} \rightarrow \nu_{i} e^{-} e^{+}}}{\mathrm{d} x}=-a^{\prime} \frac{G_{F}^{2}}{192 \pi^{3}} k_{1}^{6},
\end{gathered}
$$

with

$$
\begin{array}{cl}
a_{\mathrm{CG}}=\frac{1}{14} \delta_{i}^{3}, & a_{\mathrm{CG}}^{\prime}=\frac{25}{448} \delta_{i}^{3}, \\
a_{\mathrm{BL}, \mathrm{I}}=\frac{17}{420} \delta_{i}^{3}, & a_{\mathrm{BL}, \mathrm{I}}^{\prime}=\frac{11}{336} \delta_{i}^{3}, \\
a_{\mathrm{BL}, \mathrm{II}}=\frac{2}{35} \delta_{i}^{3}, & a_{\mathrm{BL}, \mathrm{II}}^{\prime}=\frac{5}{112} \delta_{i}^{3},
\end{array}
$$

for the model of Cohen and Glashow [26], and models I and II of Bezrukov and Lee [17]. In regard to the models of Bezrukov and Lee [see also Eq. (67)], we recall that they correspond to (i) using superluminal spin sums for all particles, so $\sigma_{i}=\sigma_{f}=1$; (ii) setting $n_{s}=1$ for the number of spin states for the neutrino; (iii) considering the emitted electron to be Lorentz invariant, hence $\delta_{f}=0$; and (iv) using $\delta_{\text {int }}=0$ for model I and $\delta_{\text {int }}=\delta_{i}$ for model II. In this case too, $\sin ^{2}\left(\theta_{W}\right)=1 / 4$ is used. We thus confirm all known results from Refs. [17,26].

Under the inclusion of a conceivably nonvanishing parameter $\delta_{e} \neq 0$, the results generalize to the form

$$
\begin{aligned}
& a_{\mathrm{CG}}=\frac{1}{14}\left(\delta_{i}-\delta_{e}\right)\left[\left(\delta_{i}-\delta_{e}\right)^{2}+\frac{5}{3} \delta_{e}\left(\delta_{i}-\delta_{e}\right)+\frac{7}{15} \delta_{e}^{2}\right], \\
& a_{\mathrm{CG}}^{\prime}=\frac{25}{448}\left(\delta_{i}-\delta_{e}\right)\left[\left(\delta_{i}-\delta_{e}\right)^{2}+\frac{112}{75} \delta_{e}\left(\delta_{i}-\delta_{e}\right)+\frac{32}{75} \delta_{e}^{2}\right],
\end{aligned}
$$

$$
\begin{aligned}
& a_{\mathrm{BL}}=\frac{17}{420}\left(\delta_{i}-\delta_{e}\right)\left[\left(\delta_{i}-\delta_{e}\right)^{2}+\frac{7}{17} \delta_{\mathrm{int}}^{2}\right], \\
& a_{\mathrm{BL}}^{\prime}=\frac{11}{336}\left(\delta_{i}-\delta_{e}\right)\left[\left(\delta_{i}-\delta_{e}\right)^{2}+\frac{4}{11} \delta_{\mathrm{int}}^{2}\right],
\end{aligned}
$$


where one would set $\delta_{\text {int }}=\delta_{i}+\delta_{e}$ in a gauge-invariant model (see the Appendixes).

Just to achieve full clarification, we remark that the interpolation between $a_{\mathrm{BL}, \mathrm{I}} \rightarrow a_{\mathrm{BL}, \mathrm{II}}$ [see Eqs. (73b) and (73c)], consistent with Eq. (76), can be traced to the algebraic identity

$$
\frac{17}{420} \times\left(1+\frac{7}{17}\right)=\frac{2}{35} \quad\left[a_{\mathrm{BL}, \mathrm{I}} \rightarrow a_{\mathrm{BL}, \mathrm{II}}\right] .
$$

The interpolation between $a_{\mathrm{BL}, \mathrm{I}}^{\prime} \rightarrow a_{\mathrm{BL}, \mathrm{II}}^{\prime}$ [see Eqs. (73b) and (73c)], consistent with Eq. (77), can be traced to the algebraic identity

$$
\frac{11}{336} \times\left(1+\frac{4}{11}\right)=\frac{5}{112} \quad\left[a_{\mathrm{BL}, \mathrm{I}}^{\prime} \rightarrow a_{\mathrm{BL}, \mathrm{II}}^{\prime}\right],
$$

which we would also like to indicate, for full clarification.

Note that the coefficients proportional to $\delta_{e}\left(\delta_{i}-\delta_{e}\right)$ vanish in the model proposed by Bezrukov and Lee [17]. Turning to the case of "slower" superluminal neutrino pair creation by emission from a "faster" superluminal neutrino, i.e., $\psi_{f}=\nu_{f}=\nu_{f}^{(m)}$, we again parametrize the total decay width and differential energy loss as

$$
\begin{gathered}
\Gamma_{\nu_{i} \rightarrow \nu_{i} \nu_{f} \bar{\nu}_{f}}=b \frac{G_{F}^{2}}{192 \pi^{3}} k_{1}^{5}, \\
\frac{\mathrm{d} E_{\nu_{i} \rightarrow \nu_{i} \nu_{f} \bar{\nu}_{f}}}{\mathrm{~d} x}=-b^{\prime} \frac{G_{F}^{2}}{192 \pi^{3}} k_{1}^{6} .
\end{gathered}
$$

In this case too, we can consider the prescription of Ref. [26] for the spin sums and the interaction Lagrangian with $v_{\text {int }}=0$. Then we find

$$
\begin{aligned}
& b_{\mathrm{CG}}=\frac{1}{7}\left(\delta_{i}-\delta_{f}\right)\left[\left(\delta_{i}-\delta_{f}\right)^{2}+\frac{5}{3} \delta_{f}\left(\delta_{i}-\delta_{f}\right)+\frac{7}{15} \delta_{f}^{2}\right], \\
& b_{\mathrm{CG}}^{\prime}=\frac{25}{224}\left(\delta_{i}-\delta_{f}\right)\left[\left(\delta_{i}-\delta_{f}\right)^{2}+\frac{112}{75} \delta_{f}\left(\delta_{i}-\delta_{f}\right)+\frac{32}{75} \delta_{f}^{2}\right] .
\end{aligned}
$$

These results are applicable for $\delta_{i}>\delta_{f}$, as explained above. (Otherwise, the available phase space for the outgoing particles vanishes.) We have checked that the $\delta_{f} \rightarrow 0$ limit corresponds to the previous result for $\psi_{f}=e$ after accounting for the different factors of $f_{e}, c_{A}, c_{V}$ as well as the number of spin states $n_{s}$, which we take as $n_{s}=2$ for the model of Cohen and Glashow, and as $n_{s}=1$ for the models of Bezrukov and Lee, in accordance with the prescriptions used in Refs. [17,26].

We can also insert the spin sum prescription from Bezrukov and Lee [17], Eq. (22), and use the pseudo-metric $\tilde{g}_{\lambda \sigma}\left(v_{\text {int }}\right)$ given in Eq. (17) with $\delta_{\text {int }}=$ $v_{\text {int }}^{2}-1$ left as a free parameter, in the interaction Lagrangian (18). In this case we obtain

$$
\begin{aligned}
& b_{\mathrm{BL}}=\frac{17}{210}\left(\delta_{i}-\delta_{f}\right)\left[\left(\delta_{i}-\delta_{f}\right)^{2}+\frac{7}{17} \delta_{\mathrm{int}}^{2}\right], \\
& b_{\mathrm{BL}}^{\prime}=\frac{11}{168}\left(\delta_{i}-\delta_{f}\right)\left[\left(\delta_{i}-\delta_{f}\right)^{2}+\frac{4}{11} \delta_{\mathrm{int}}^{2}\right] .
\end{aligned}
$$

Better (but not full) compliance with $S U(2)_{L}$ gauge invariance is ensured by setting $\delta_{\text {int }}=\delta_{i}+\delta_{f}$ (see Appendixes A and B).

Finally, we offer a comment on the LPCR process assuming that also the electron is slightly superluminal. Assuming that $\delta_{e}$ is of the same order of magnitude as $\delta_{\nu}$ and $\delta_{\text {int }}$, Eqs. (67) and (70) demonstrate that the results presented above in Eqs. (82) and (83) (valid to leading order in the small $\delta$ 's) for the NPCR process are only modified with respect to LPCR due to the different values of $f_{e}$ [see Eq. (19)] and the couplings $c_{A}$ and $c_{V}$ [see Eq. (20)]. Accounting for these differences simply amounts to dividing the results of Eqs. (82) and (83) by a factor of 2 . We note that after taking this extra factor of $1 / 2$ into account, the $\delta_{f} \rightarrow 0$ limit of Eqs. (82) and (83) indeed reproduces the results of Eq. (73) with $\delta_{\text {int }}$ chosen appropriately: $\delta_{\text {int }}=0$ for the Cohen-Glashow model, and model I of Bezrukov and Lee, while $\delta_{\text {int }}=\delta_{i}+\delta_{f} \rightarrow \delta_{i}$ for model II of Ref. [17].

\section{B. Interpretation of the results}

Let us try to interpret the results presented in Sec. IVA, both qualitatively and quantitatively.

The first point to address is the fractional energy loss during a NPCR decay process. The double-differential energy loss with respect to energy and time, due to VPE or neutrino splitting, is

$$
\mathrm{d} E_{1}=\left(E_{1}-E_{3}\right) \frac{\mathrm{d} \Gamma}{\mathrm{d} E_{3}} \mathrm{~d} t=\left(E_{1}-E_{3}\right) \frac{\mathrm{d} \Gamma}{\mathrm{d} E_{3}} \frac{\mathrm{d} x}{c},
$$

where we restore the factor of $c$ for clarity. One then obtains, in natural units,

$$
\frac{\mathrm{d} E_{1}}{\mathrm{~d} x}=\int_{0}^{E_{1}}\left(E_{1}-E_{3}\right) \frac{\mathrm{d} \Gamma}{\mathrm{d} E_{3}} \mathrm{~d} E_{3},
$$

but the expression of the right-hand side of this formula has the alternative interpretation as the mean energy loss during a decay event. Hence, the average fractional energy loss during a decay event can be evaluated as

$$
f=\frac{1}{E_{1} \Gamma} \frac{\mathrm{d} E_{1}}{\mathrm{~d} x}
$$


which is a constant for all processes described by Eqs. (71)-(83). For LPCR, in the Cohen-Glashow formulation [26], one obtains $f=(25 / 224) /(1 / 7)=$ $25 / 32=0.78$, which explains the remark made in Refs. $[8,14]$ regarding an average $78 \%$ energy loss during each single vacuum pair emission event.

For the term proportional to $\left(\delta_{i}-\delta_{f}\right)^{3}$ in NPCR, still within the Cohen-Glashow formulation [26], one obtains the same ratio $f=(25 / 112) /(2 / 7)=0.78$, while for the term proportional to $\left(\delta_{i}-\delta_{f}\right) \delta_{f}^{2}$, the result is $f=7 / 10=0.7$. For the model II of Bezrukov and Lee [17], one obtains ratios of $f=55 / 68=0.81$ [for the $\left(\delta_{i}-\delta_{f}\right)^{3}$ term] and $f=5 / 7=0.71$ [for the $\left(\delta_{i}-\delta_{f}\right) \delta_{\text {int }}^{2}$ term]. At variance with these results, in Ref. [8], an equipartition of energy among the products of the neutrino splitting is assumed, based on a Planck-scale generated, dimension-six operator.

Let us investigate if the NPCR process could still generate a substantial contribution to neutrino energy losses on astrophysical timescales, given the tight constraints on the difference between Lorentz-violating parameters coming from short-baseline [19] and "extremely long-baseline" experiments [21].

The model preferred in Ref. [8] concerns a dimensionsix operator which leads to a decay rate that has the same functional dependence as Eq. (80), but with the $b$ parameter replaced by an expression proportional to

$$
b \propto\left(\frac{k_{1}}{M_{\mathrm{Pl}}}\right)^{6}
$$

corresponding to a replacement of the term $\left(\delta_{i}-\delta_{f}\right) \delta_{f}^{2}$ by an expression proportional to

$$
\left(\delta_{i}-\delta_{f}\right) \delta_{f}^{2} \propto\left(\frac{k_{1}}{M_{\mathrm{Pl}}}\right)^{6} .
$$

(This formula should be compared to a potential energy dependence of Lorentz-violating parameters, as envisaged in Ref. [36].)

The surprising conclusion is as follows. Even at the fantastically small differential Lorentz violations of $\delta_{i}-\delta_{f}<7.4 \times 10^{-27}$ (see Ref. [21]), the NPCR process can still substantially contribute to the energy loss of neutrinos on astrophysical scales, if we use $\delta_{f} \sim$ $1.0 \times 10^{-20}[8,14]$. Namely, at an energy of $10 \mathrm{PeV}$, which according to Refs. $[8,14]$ is commensurate with a cutoff of the astrophysical neutrino spectrum at about $2 \mathrm{PeV}$ (arriving on Earth), our process induces a decay rate, and an energy loss rate, commensurate with a Planck-scale, dimension-six operator with a large numerical coefficient $\kappa_{2}=258$ (in the conventions of Ref. [8]). For $\delta_{i}-\delta_{f} \lesssim$ $6 \times 10^{-22}$ (see Ref. [20]), a Planck-scale, dimension-six operator with a large numerical coefficient of $\kappa_{2}=9200$ would be required to lead to a comparable effect at the quoted energy of $10 \mathrm{PeV}$. For comparison, we note that in order to explain a putative cutoff of cosmic neutrinos at energy $2 \mathrm{PeV}$, a value of $\kappa_{2}=7800$ is otherwise required [8]. This observation illustrates that the final evaluation of the relevance of the NPCR process could depend on the clarification of the precise location, and the physical mechanism behind the conjectured cutoff of the highenergy cosmic neutrino spectrum.

A semiquantitative observation is of interest. Namely, a signature of the NPCR process would be a departure from a $(1: 1: 1)_{\oplus}$ equipartition of neutrino flavors arriving on Earth, due to a decay of all neutrino mass eigenstates except the slowest one, above a certain energy scale where the decay channel becomes numerically relevant. (This is perhaps not so evident for the dimension-six operator verified in Ref. [8], but evident for our scenario studied here. The general idea that neutrino decays could alter the flavor composition arriving on Earth has been formulated in Ref. [37].) An analysis of neutrinos above $35 \mathrm{TeV}$ arriving at IceCube [38] (see also Refs. [39-41]) is statistically compatible with an equal flavor distribution $(1: 1: 1)_{\oplus}$ on Earth, but in the caption of Fig. 3 of Ref. [38], it is explicitly stated that the best-fit composition at Earth is $(0: 1 / 5: 4 / 5)_{\oplus}$. In the caption of Fig. 5 of the recent work [42], the best-fit composition is given as $(0: 0.21: 0.79)_{\oplus}$. Let us have a look at the structure of the PMNS matrix, which is given as follows:

$$
U=\left(\begin{array}{ccc}
U_{e 1} & U_{e 2} & U_{e 3} \\
U_{\mu 1} & U_{\mu 2} & U_{\mu 3} \\
U_{\tau 1} & U_{\tau 2} & U_{\tau 3}
\end{array}\right) .
$$

Modulus-wise, one has very large mixing [see Eq. (2.2) of Ref. [43] ],

$$
\begin{aligned}
|U| & =\left(\begin{array}{lll}
\left|U_{e 1}\right| & \left|U_{e 2}\right| & \left|U_{e 3}\right| \\
\left|U_{\mu 1}\right| & \left|U_{\mu 2}\right| & \left|U_{\mu 3}\right| \\
\left|U_{\tau 1}\right| & \left|U_{\tau 2}\right| & \left|U_{\tau 3}\right|
\end{array}\right) \\
& =\left(\begin{array}{lll}
0.797 \ldots 0.842 & 0.518 \ldots 0.585 & 0.143 \ldots 0.156 \\
0.235 \ldots 0.484 & 0.458 \ldots 0.671 & 0.647 \ldots 0.781 \\
0.304 \ldots 0.531 & 0.497 \ldots 0.699 & 0.607 \ldots 0.747
\end{array}\right) .
\end{aligned}
$$

The "second" mass eigenstate thus has the roughly equal flavor decomposition

$$
\nu_{2}^{(m)}=U_{e 2}^{*} \nu_{e}^{(f)}+U_{\mu 2}^{*} \nu_{\mu}^{(f)}+U_{\tau 2}^{*} \nu_{\tau}^{(f)},
$$

where $\left|U_{e 2}^{*}\right| \approx\left|U_{\mu 2}^{*}\right| \approx\left|U_{\tau 2}^{*}\right| \approx 1 / \sqrt{3}$ (within numerical uncertainty), while mass eigenstate "number 3 " leans more toward a higher $\mu$-neutrino and $\tau$-neutrino content, consistent with the trend of the data reported in Fig. 3 of 


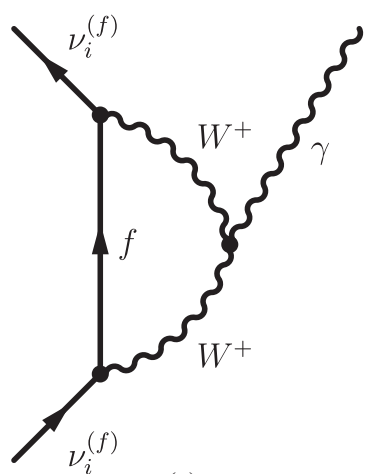

(a)

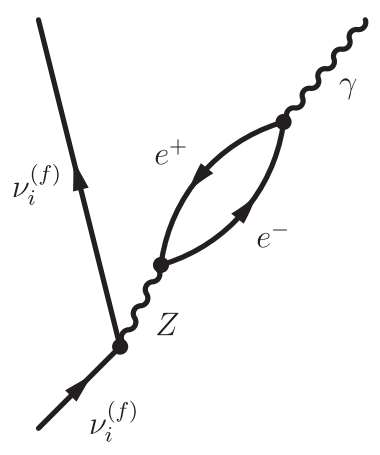

(b)

FIG. 3. In genuine Cerenkov radiation $\nu \rightarrow \nu+\gamma$ (for superluminal neutrinos), the photon is emitted from a $W$ loop (a) or from a vacuum-polarization insertion into the $Z$ propagator (b); the emission takes place from a flavor eigenstate $\left|\nu_{i}^{(f)}\right\rangle$. The photon emission thus involves an extra factor $\alpha_{\mathrm{QED}} \approx 1 / 137.036$ at the $W$-photon vertex as compared to LPCR and NPCR.

Ref. [38]. In principle, one could thus speculate about mass eigenstate number 3 serving as the "slowest" mass eigenstate, which thus would be the only one not affected by neutrino splitting. However, at present, this observation does not go beyond the status of pure speculation, in the sense of "reading the tea leaves." In particular, the parameters required for the NPCR process, as formulated here, are constrained to a kinematic region incompatible with a conjectured caused cutoff of all neutrino mass eigenstates except the slowest one, at a comparatively low energy of only $35 \mathrm{TeV}$.

Likewise, it is instructive to compare the consequences of the neutrino splitting and its signatures to those discussed in Ref. [44], where the authors present calculations for the flavor ratio of IceCube in the high-energy region, given the putative presence of a $\nu \rightarrow 3 \nu$ neutrino splitting mechanism in the high-energy region, albeit a different one as compared to the one discussed here, namely, mediated by a second Higgs doublet (box diagrams). The authors of Ref. [44] consider a model based on the symmetry group $S U(3)_{c} \times S U(2)_{L} \times U(1)_{Y} \times Z_{2}$, which implies that for any usual Standard Model fermion, one has one electroweak singlet right-handed neutrino. The model is referred to as the 2 2HDM (neutrino-two-Higgs doublet model).

In the text following Eq. (4.3) of Ref. [44], the authors present predictions for neutrino flavor ratios, based on additional assumptions on the production mechanism and the mass hierarchy. They ascertain that, for normal mass hierarchy, neutrinos coming from pion decay split, according to $\nu \rightarrow 3 \nu$, to produce flavor ratios of daughter neutrinos in the flavor basis as $\phi_{e}: \phi_{\mu}: \phi_{\tau} \approx$ $2: 1: 1$. For neutrinos coming from muon damped sources, still with normal mass hierarchy, the prediction is $\phi_{e}: \phi_{\mu}: \phi_{\tau} \approx 1.8: 1: 1$. For neutrinos from neutron decay, the splitting is $\phi_{e}: \phi_{\mu}: \phi_{\tau} \approx 3: 1: 1$.

None of the predictions presented in Ref. [44] is consistent with a flavor ratio that would correspond to exactly one of the mass eigenstates of the PMNS matrix; the latter would otherwise be predicted by the Lorentzviolation mediated neutrino splitting model. Namely, as already stressed, the superluminal model would predict that only one mass eigenstate survives. So, even though questions regarding the mass hierarchy of the neutrino mass eigenstates have not been conclusively addressed, we can say that the model discussed in our work and the one discussed in Ref. [44] have distinctly different signatures.

\section{CONCLUSIONS}

Let us summarize the most important findings reported here.

(i) (i) Three decay processes have been identified in Ref. [26] which become kinematically possible for an incoming, superluminal, Lorentz-violating neutrino: LPCR [Eq. (8)], NPCR [Eq. (9)], and the process $\nu \rightarrow \nu+\gamma$; the latter constitutes genuine Cerenkov radiation in vacuum (see Fig. 3). For $\nu \rightarrow \nu+\gamma$, the photon is emitted from a $W$ loop or from a vacuumpolarization correction to the $Z$ propagator, and thus involves an extra factor $\alpha_{\mathrm{QED}} \approx 1 / 137.036$. This process is thus parametrically suppressed. Here, we show that the NPCR process is not parametrically suppressed in comparison to LPCR. Furthermore, the threshold for NPCR is at least 6 orders of magnitude lower than for LPCR [Eq. (10)]. We assume that the velocity parameters $v_{i}$ in the dispersion relation (11) for the three mass eigenstates are not all identical. While the relative differences among Lorentzviolating parameters for the neutrino mass and flavor eigenstates are otherwise tightly constrained [19-21], our assumption is supported by the fact that the corresponding mass terms $m_{i}$ in Eq. (11) also are different from each other.

(ii) We here confirm the results obtained for LPCR obtained in Refs. [17,26], including the model dependence derived in Ref. [17]. This also reaffirms the validity of the astrophysical bounds on the Lorentzviolating parameters, derived in Refs. [8,14], based on dimension-four operators. Our expressions for the NPCR process are parametrically of the same order as for LPCR, but the overall coefficients are larger by a factor 4 or 5 . We should also point out the somewhat unexpected terms in the NPCR decay rates reported in Eqs. (82) and (83), for Lorentz-violating neutrinos, proportional to $\left(\delta_{i}-\delta_{f}\right) \delta_{f}^{2}$ instead of $\left(\delta_{i}-\delta_{f}\right)^{3}$. We also derive a few more pieces of information, e.g., the departure from the equipartition of energy between the decay products of neutrino splitting, with the incoming neutrino being shown to lose about $(75 \pm 5) \%$ of its energy during NPCR decay. The structure of the dimension-four NPCR operators derived here also 
raises a pertinent question regarding the possible presence of terms proportional to $\Theta\left(\delta_{i}-\delta_{f}\right)$ (where $\Theta$ is the Heaviside step function) in the dimension-five and dimension-six operators, which were derived from Planck-scale physics (see Ref. [8]).

(iii) If, in the future, hypothetically, the high-energy behavior of neutrinos should be confirmed to be superluminal and Lorentz violating, but with exceedingly small parameters, then our results will help in the modeling of the influence of the LPCR process on intergalactic neutrino propagation.

Any statement beyond the above observations would require an elaborate Monte Carlo simulation of astrophysical data (see Refs. $[8,14]$ ), which is beyond the scope of the current paper. A general picture emerges from the analysis of LPCR and NPCR for high-energy incoming, superluminal neutrinos: namely, at high energy, even very tiny parameters $\delta$ lead to a high virtuality $E^{2}-\vec{p}^{2}=$ $\left(v^{2}-1\right) \vec{p}^{2} \approx \delta \times E^{2}$, which grows with the energy. A high virtuality implies that various pair production processes become kinematically possible in the high-energy domain. This observation leads to the very strict bounds on the Lorentz-violating parameters and puts very tight constraints on the Lorentz-violating models. Indeed, the LPCR and NPCR process, as well as vacuum Cerenkov radiation, imply very tight restrictions on the available parameter space for Lorentz violation in the high-energy neutrino sector.

\section{ACKNOWLEDGMENTS}

The authors would like to mention insight discussions with, and advice from, A. de Gouvea. Support from the National Science Foundation (Grant No. PHY-1710856) is gratefully acknowledged. This work was also supported by the ÚNKP-17-3 New National Excellence Program of the Ministry of Human Capacities of Hungary and by Grant No. K125105 of the National Research, Development and Innovation Fund in Hungary.

\section{APPENDIX A: Generalized Dirac Equations}

It is instructive to consider the derivation of the Lorentzviolating dispersion relation (11) on the basis of a generalized Dirac equation, as well as the statement made after Eq. (18), which concerns the fact that the Lagrangian in Eq. (18), for $v_{\text {int }}=v_{i}$, can be considered as an $S U(2)_{L}$-gauge-invariant Lagrangian, whereas for $v_{\text {int }}=1$ $\left(v_{\text {int }}=c\right)$, or $\delta_{\text {int }}=0$, it is not gauge invariant. In order to proceed with the proof, let us denote by

$$
g_{\mu \nu}=\operatorname{diag}(1,-1,-1,-1)
$$

the flat-space, standard space-time metric, and by $\tilde{g}_{\mu \nu}$ a generalized pseudo-metric of constant coefficients, which parametrizes the Lorentz violation. It can take the form [see Eq. (17)]

$$
\tilde{g}_{\mu \nu}\left(v^{2}\right)=\operatorname{diag}\left(1,-v^{2},-v^{2},-v^{2}\right),
$$

where we note the square of the velocity, but the formalism outlined below is more general. We define the generalized Dirac matrices [cf., Refs. [45-50]]

$$
\tilde{\gamma}_{\mu}=e_{\mu}^{A} \gamma_{A},
$$

where the Einstein summation convention is used, and $\gamma^{A}$ with $A=0,1,2,3$ are the ordinary Dirac $\gamma$ matrices, while the $e_{\mu}^{A}$ take the role of the so-called "vierbein" in general relativity, with the property

$$
\tilde{g}_{\mu \nu}=e_{\mu}^{A} g_{A B} e_{\nu}^{B}=e_{\mu}^{A} e_{\nu A} .
$$

This implies that the vierbein takes the role of the square root of the metric [46]. Capital latin indices can be raised with the flat-space metric $g^{A B}$. One can then easily show that

$$
\left\{\tilde{\gamma}_{\mu}, \tilde{\gamma}_{\nu}\right\}=e_{\mu}^{A} e_{\nu}^{B}\left\{\gamma_{A}, \gamma_{B}\right\}=e_{\mu}^{A} e_{\nu}^{B}\left(2 g_{A B}\right)=2 \tilde{g}_{\mu \nu} .
$$

The analogy to the formalism of general relativity implies that $\tilde{g}_{\mu \nu}$ takes the role of a modified Lorentz "metric," but without curvature (because we assume that the coefficients are constant). The word "metric" should be understood with a grain of salt (hence the apostrophes), because it does not constitute a space-time metric in the sense of general relativity, that is used to measure spacetime intervals, but rather, a mathematical object used to parametrize the dispersion relation of a Lorentz-violating particle. Because of the lack of curvature, the pseudometric $\tilde{g}_{\mu \nu}$ is still characterizing a flat "space-time." (For a truly curved space, the notation $\bar{g}_{\mu \nu}$ has been proposed in Refs. [45,46] in order to distinguish the curved-space quantities from the flat-space ones.) The $\tilde{\gamma}_{\mu}$ are thus intermediate in between the usual flat-space Dirac matrices, and the curved-space matrices, for which the notation $\bar{g}_{\mu \nu}$ has been proposed in Refs. [45-50].

For a modified pseudo-metric of the form (A2), one can choose the vierbein coefficients as

$e_{0}^{0}=1, \quad e_{i}^{0}=e_{0}^{i}=0, \quad e_{j}^{i}=v \delta_{j}^{i}, \quad i, j=1,2,3$.

The modified Dirac equation describing the Lorentz violation can then be written as

$$
\left(\mathrm{i} \tilde{\gamma}_{\mu} \partial^{\mu}-m\right) \psi=0 .
$$

We here suppress the chirality projectors and assume that $\psi$ is a left-handed field. One can multiply from the left by the operator $\left[\mathrm{i} \tilde{\gamma}_{\nu} \partial^{\nu}+m\right]$, and use the operator identity 


$$
\left(\mathrm{i} \tilde{\gamma}_{\nu} \partial^{\nu}+m\right)\left(\mathrm{i} \tilde{\gamma}_{\mu} \partial^{\mu}-m\right)=-\tilde{g}_{\mu \nu} \partial^{\mu} \partial^{\nu}-m^{2} .
$$

For the pseudo-metric (A2), one can use the identity

$$
-\tilde{g}_{\mu \nu} \partial^{\mu} \partial^{\nu}-m^{2}=E^{2}-v^{2} \vec{p}^{2}-m^{2},
$$

where $E$ is the energy and $\vec{p}$ is the momentum operator. This leads to the dispersion relation,

$$
E= \pm \sqrt{\vec{p}^{2} v^{2}+m^{2}}
$$

which is equivalent to Eq. (11). We here note, though, that Eq. (11) has no gauge structure; it is formulated for free particles.

\section{APPENDIX B: Gauge Invariance of the Models}

For a full clarification of the models used in the current investigation, and in order to avoid misunderstandings, a number of remarks on the gauge (non)invariance of the interaction Lagrangian (18) are in order. Of course, the interaction Lagrangian (18) in itself of course does not describe a gauge-invariant theory, but merely constitutes the low-energy limit of the full electroweak theory. It is still applicable for the description of the decay and energy loss processes analyzed in the current paper [see also the remarks in the text preceding Eq. (18)].

Furthermore, the velocity parameter $v_{\text {int }}$ that enters Eq. (18) of course depends on the details of the gaugeinvariant theory that one started from, in the derivation of the effective "low-energy" interaction given in Eq. (18).

We should mention that questions related to the gauge (non)invariance of the models have recently been analyzed in detail, in a separate paper [27]. We would like to provide a summary here. In the model used by Cohen and Glashow [26], which corresponds to model I used by Bezrukov and Lee [17], and also corresponds to the interaction Lagrangian (18) with $v_{\text {int }}=1$, it cannot be overemphasized that $S U(2)_{L}$ gauge invariance is manifestly broken. In fact, model I used by Bezrukov and Lee [17] is obtained if one postulates that the Lorentz-breaking term introduced into the free Dirac equation for the Lorentz-violating neutrinos is not "gauged," i.e., retains the partial derivative as opposed to the covariant derivative in the neutrino sector. In this case, the interaction Lagrangian is unaltered in comparison to the Fermi theory, while the free Lagrangian of the neutrinos acquires a Lorentz-violating term. In order to put things into perspective, one should note that the gauge dependence in model I enters only at the perturbative level, i.e., on the same level as the Lorentz-violating operator itself enters the Lagrangian [27]. In order to verify that model I is based on reasonable assumptions, one can point to the observations made in the papers of Nielsen et al. [51-58], where the authors (in a somewhat different context) observe the emergence of gauge-symmetry-breaking terms, upon the introduction of (initially) spontaneous Lorentz- (but not gauge-) symmetry breaking. In this context, we note that the three-photon vertex and the two-fermion, two-photon interaction in the Lagrangian given in Eq. (3) of Ref. [57], which are initially generated by spontaneous Lorentz breaking in the electromagnetic sector, break electromagnetic gauge invariance. With reference to Eqs. (9) and (10) of Ref. [1], it cannot be stressed enough that the model used by Cohen and Glashow in Ref. [26] is outside of the original formulation of the Standard Model extension [1].

Even model II used by Bezrukov and Lee [17], somewhat interestingly, is not gauge invariant with respect to the full $S U(2)_{L} \times U(1)_{Y}$ gauge theory (see Sec. IV of Ref. [27]), but follows from the full gauge theory if one reduces the gauge group to $U(1) \times U(1)_{Y}$, i.e., to a sector where only the interaction terms corresponding to the $Z$ boson and the photon are (re)diagonalized (but not the $W$ boson interaction terms). The partial retention of gauge invariance justifies, a posteriori, to a certain degree, the statement made by Bezrukov and Lee that their model II is "gauge invariant" where we note the quotation marks in the text after Eq. (4) of Ref. [17], implying that their statement should be taken cum grano salis. Again, with reference to Eqs. (9) and (10) of Ref. [1], model II used by Bezrukov and Lee [17] also is outside of the original formulation of the Standard Model extension, but implements a restricted set of symmetry groups: Namely, it reduces the Lorentz symmetry group from $S O(1,3)$ to $S O(3)$, in view of the spatially isotropic Lorentz violation, and the electroweak gauge group from $S U(2)_{L} \times U(1)_{Y}$ to $U(1) \times U(1)_{Y}$. Model II thus constrains Lorentz-breaking and gaugesymmetry-breaking parameters within the given restricted symmetry groups.

As a last point, we mention that, as pointed out in Sec. V of Ref. [27], there might actually be a possibility to formulate a Lorentz-violating theory, which fully preserves $S U(2)_{L} \times U(1)_{Y}$ gauge invariance and still allows for the LPCR and NPCR decays. The decisive idea is to postulate Lorentz-violating parameters which depend on the flavor (see Sec. V of Ref. [27]). We recall that, if all left-handed charged fermions and all neutrinos are grouped together in $S U(2)_{L}$ multiplets and if there is uniform Lorentz violation over all generations (flavors), then both NPCR and LPCR are kinematically forbidden. However, if the Lorentzviolating parameters are different among the fermion flavors, then the processes become kinematically allowed, and under the given assumptions, with a fully $S U(2)_{L} \times$ $U(1)_{Y}$ covariant coupling, the form of the interaction Lagrangians is uniquely determined. Details on further calculations based on these models, including additional proofs regarding the gauge invariance with respect to the particular choice for the gauge boson propagator, will be published elsewhere [59]. 
[1] D. Colladay and V. A. Kostelecký, Lorentz-violating extension of the standard model, Phys. Rev. D 58, 116002 (1998).

[2] V. A. Kostelecky and M. Mewes, Electrodynamics with Lorentz-violating operators of arbitrary dimension, Phys. Rev. D 80, 015020 (2009).

[3] J. S. Diaz, V. A. Kostelecky, and M. Mewes, Perturbative Lorentz and CPT violation for neutrino and antineutrino oscillations, Phys. Rev. D 80, 076007 (2009).

[4] V. A. Kostelecky and M. Mewes, Neutrinos with Lorentzviolating operators of arbitrary dimension, Phys. Rev. D 85, 096005 (2012).

[5] J. S. Diaz, V. A. Kostelecky, and M. Mewes, Testing relativity with high-energy astrophysical neutrinos, Phys. Rev. D 89, 043005 (2014).

[6] J. Diaz, Neutrinos as probes of Lorentz invariance, Adv. High Energy Phys. 2014, 962410 (2014).

[7] J. D. Tasson, What do we know about Lorentz invariance, Rep. Prog. Phys. 77, 062901 (2014).

[8] F. W. Stecker, S. T. Scully, S. Liberati, and D. Mattingly, Searching for traces of Planck-scale physics with high energy neutrinos, Phys. Rev. D 91, 045009 (2015).

[9] S. Liberati, Lorentz symmetry breaking: Phenomenology and constraints, J. Phys. Conf. Ser. 631, 012011 (2015).

[10] M. G. Aartsen et al. (IceCube Collaboration), First Observation of PeV-Energy Neutrinos with IceCube, Phys. Rev. Lett. 111, 021103 (2013).

[11] M. G. Aartsen et al. (IceCube Collaboration), Observation of High-Energy Astrophysical Neutrinos in Three Years of IceCube Data, Phys. Rev. Lett. 113, 101101 (2014).

[12] O. Botner, Proceedings of the IceCube Neutrino Observatory: Present and Future, 2015 IceCube Particle Astrophysics Symposium, Madison, WI (2015).

[13] F. W. Stecker, Limiting superluminal electron and neutrino velocities using the 2010 Crab Nebula flare and the IceCube PeV neutrino events, Astropart. Phys. 56, 16 (2014).

[14] F. W. Stecker and S. T. Scully, Propagation of superluminal PeV IceCube neutrinos: A high energy spectral cutoff of new constraints on Lorentz invariance violation, Phys. Rev. D 90, 043012 (2014).

[15] P. B. Pal and L. Wolfenstein, Radiative decays of massive neutrinos, Phys. Rev. D 25, 766 (1982).

[16] S. L. Glashow, J. Iliopoulos, and L. Maiani, Weak interactions with lepton-hadron symmetry, Phys. Rev. D 2, 1285 (1970).

[17] F. Bezrukov and H. M. Lee, Model dependence of the bremsstrahlung effects from the superluminal neutrino at OPERA, Phys. Rev. D 85, 031901(R) (2012).

[18] L. Maccione, S. Liberati, and D. M. Mattingly, Violations of Lorentz invariance in the neutrino sector: An improved analysis of anomalous threshold constraints, J. Cosmol. Astropart. Phys. 03 (2013) 039.

[19] A. A. Aguilar-Arevalo et al. (MiniBooNE Collaboration), Test of Lorentz and CPT violation with short baseline neutrino oscillation excesses, Phys. Lett. B 718, 1303 (2013).

[20] S. Coleman and S. L. Glashow, High-energy tests of Lorentz invariance, Phys. Rev. D 59, 116008 (1999).

[21] R. Abbasi et al. (IceCube Collaboration), Search for a Lorentz-violating sidereal signal with atmospheric neutrinos in IceCube, Phys. Rev. D 82, 112003 (2010).
[22] D. M. Mattingly, L. Maccione, M. Galaverni, S. Liberati, and G. Sigl, Possible cosmogenic neutrino constraints on Planck-scale Lorentz violation, J. Cosmol. Astropart. Phys. 02 (2010) 007.

[23] J. M. Carmona and J. L. Cortes, Constraints from neutrino decay on superluminal velocities, arXiv:1110.0430.

[24] E. Ciuffoli, J. Evslin, X. Bi, and X. Zhang, Neutrino splitting and density-dependent dispersion relations, ISRN High Energy Phys. 2012, 436580 (2012).

[25] Y. Huo, T. Li, Y. Liao, D. V. Nanopoulos, and Y. Qi, Constraints on neutrino velocities revisited, Phys. Rev. D 85, 034022 (2012).

[26] A. G. Cohen and S. L. Glashow, Pair Creation Constrains Superluminal Neutrino Propagation, Phys. Rev. Lett. 107, 181803 (2011).

[27] U. D. Jentschura, I. Nándori, and G. Somogyi, Lorentz Breaking and $S U(2)_{L} \times U(1)_{Y}$ gauge invariance for neutrino decays, arXiv:1908.01389.

[28] U. D. Jentschura, I. Nándori, and R. Ehrlich, Calculation of the decay rate of tachyonic neutrinos against chargedlepton-pair and neutrino-pair Cerenkov radiation, J. Phys. G 44, 105201 (2017).

[29] X.-J. Bi, P.-F. Yin, Z.-H. Yu, and Qiang Yuan, Constraints and Tests of the OPERA Superluminal Neutrinos, Phys. Rev. Lett. 107, 241802 (2011).

[30] B. Altschul, Astrophysical limits on Lorentz violation for all charged species, Astropart. Phys. 28, 380 (2007).

[31] D. Colladay and V. A. Kostelecky, Cross sections and Lorentz violation, Phys. Lett. B 511, 209 (2001).

[32] E. Byckling and K. Kajantie, Particle Kinematics (J. Wiley \& Sons, New York, 1973).

[33] G. Somogyi, A subtraction scheme for computing QCD jet cross sections at NNLO: Integrating the doubly unresolved subtraction terms, J. High Energy Phys. 04 (2013) 010.

[34] V. Del Duca, G. Somogyi, and Z. Trocsanyi, Integration of collinear-type doubly unresolved counterterms in NNLO jet cross sections, J. High Energy Phys. 06 (2013) 079.

[35] S. R. Coleman and S. L. Glashow, Cosmic ray and neutrino tests of special relativity, Phys. Lett. B 405, 249 (1997).

[36] J. Alexandre, J. Ellis, and N.E. Mavromatos, On the possibility of superluminal neutrino propagation, Phys. Lett. B 706, 456 (2012).

[37] J. F. Beacom, N. F. Bell, D. Hooper, S. Pakvasa, and T. J. Weiler, Decay of High-Energy Astrophysical Neutrinos, Phys. Rev. Lett. 90, 181301 (2003).

[38] M. G. Aartsen et al. (IceCube Collaboration), Flavor Ratio of Astrophysical Neutrinos above $35 \mathrm{TeV}$ in IceCube, Phys. Rev. Lett. 114, 171102 (2015).

[39] M. Bustamante, J. F. Beacom, and W. Winter, Theoretically Palatable Flavor Combinations of Astrophysical Neutrinos, Phys. Rev. Lett. 115, 161302 (2015).

[40] C. A. Arguelles, T. Katori, and J. Salvado, Effect of New Physics in Astrophysical Neutrino Flavor, Phys. Rev. Lett. 115, 161303 (2015).

[41] S. W. Li, M. Bustamante, and J. F. Beacom, Echo Technique to Distinguish Flavors of Astrophysical Neutrinos, Phys. Rev. Lett. 122, 151101 (2019). 
[42] M. G. Aartsen et al. (IceCube Collaboration), Measurements using the inelasticity distribution of multi-TeV neutrino interactions in IceCube Phys. Rev. D 99, 032004 (2019).

[43] I. Esteban, M. C. Gonzalez-Garcia, A. Hernandez-Cabezudo, M. Maltoni, and T. Schwetz, Global analysis of three-flavour neutrino oscillations: Synergies and tensions in the determination of $\theta_{23}, \delta_{\mathrm{CP}}$, and the mass ordering, J. High Energy Phys. 01 (2019) 106..

[44] S. Mohanty and S. Sadhukhan, Explanation of IceCube spectrum with $\nu \rightarrow 3 \nu$ neutrino splitting in a $\nu 2$ HDM model, J. High Energy Phys. 10 (2018) 111.

[45] U. D. Jentschura, Gravitationally coupled Dirac equation for antimatter, Phys. Rev. A 87, 032101 (2013); Erratum, 87, 069903 (2013).

[46] U. D. Jentschura and J. H. Noble, Nonrelativistic limit of the Dirac-Schwarzschild Hamiltonian: Gravitational Zitterbewegung and gravitational spin-orbit coupling, Phys. Rev. A 88, 022121 (2013).

[47] U. D. Jentschura and J. H. Noble, Foldy-Wouthuysen transformation, scalar potentials and gravity, J. Phys. A 47, 045402 (2014).

[48] J. H. Noble and U. D. Jentschura, Ultrarelativistic decoupling transformation for generalized Dirac equations, Phys. Rev. A 92, 012101 (2015).

[49] J. H. Noble and U. D. Jentschura, Dirac Hamiltonian and Reissner-Nordström metric: Coulomb interaction in curved space-time, Phys. Rev. A 93, 032108 (2016).
[50] U. D. Jentschura, Gravitational effects in $g$ factor measurements and high-precision spectroscopy: Limits of Einstein's equivalence principle, Phys. Rev. A 98, 032508 (2018).

[51] J. L. Chkareuli, C. D. Froggatt, and H. B. Nielsen, Lorentz Invariance and Origin of Symmetries, Phys. Rev. Lett. 87, 091601 (2001).

[52] J. L. Chkareuli, C. D. Froggatt, and H. B. Nielsen, Spontaneously generated gauge invariance, Nucl. Phys. B609, 46 (2001).

[53] J.D. Bjorken, Emergent gauge bosons, arXiv:hep-th/ 0111196.

[54] A. T. Azatov and J. L. Chkareuli, Nonlinear QED and physical Lorentz invariance, Phys. Rev. D 73, 065026 (2006).

[55] J. L. Chkareuli and Z. R. Kepuladze, Nonlinear massive QED and physical Lorentz invariance, Phys. Lett. B 644, 212 (2007).

[56] J. L. Chkareuli, C. D. Froggatt, J. G. Jejeleva, and H. B. Nielsen, Constrained gauge fields from spontaneous Lorentz violation, Nucl. Phys. B796, 211 (2008).

[57] J. L. Chkareuli and J. G. Jejeleva, Spontaneous Lorentz violation: Non-Abelian gauge fields as pseudo-Goldstone vector bosons, Phys. Lett. B 659, 754 (2008).

[58] J. L. Chkareuli, C. D. Froggatt, and H. B. Nielsen, Deriving gauge symmetry and spontaneous Lorentz violation, Nucl. Phys. B821, 65 (2009).

[59] G. Somogyi, U.D. Jentschura, and I. Nándori (in preparation). 